\title{
Spin effects on neutron star fundamental-mode dynamical tides: Phenomenology and comparison to numerical simulations
}

\author{
Jan Steinhoff $\odot,{ }^{1}$ Tanja Hinderer, ${ }^{2}$ Tim Dietrich $\odot,{ }^{3,1}$ and Francois Foucart ${ }^{4}$ \\ ${ }^{1}$ Max-Planck-Institute for Gravitational Physics (Albert-Einstein-Institute), Am Mühlenberg 1, 14476 Potsdam-Golm, \\ Germany, European Union \\ ${ }^{2}$ Institute for Theoretical Physics, Utrecht University, Princetonplein 5, 3584 CC Utrecht, The Netherlands, European Union \\ ${ }^{3}$ Institut für Physik und Astronomie, Universität Potsdam, Haus 28, Karl-Liebknecht-Str. 24/25, 14476, Potsdam, Germany, European Union \\ ${ }^{4}$ Department of Physics, University of New Hampshire, 9 Library Way, Durham, New Hampshire 03824, USA
}

(Received 26 March 2021; accepted 22 July 2021; published 9 August 2021)

\begin{abstract}
Gravitational waves from neutron star binary inspirals contain information on strongly interacting matter in unexplored, extreme regimes. Extracting this requires robust theoretical models of the signatures of matter in the gravitational-wave signals due to spin and tidal effects. In fact, spins can have a significant impact on the tidal excitation of the quasinormal modes of a neutron star, which is not included in current state-of-the-art waveform models. We develop a simple approximate description that accounts for the Coriolis effect of spin on the tidal excitation of the neutron star's quadrupolar and octupolar fundamental quasinormal modes and incorporate it in the SEOBNRv4T waveform model. We show that the Coriolis effect introduces only one new interaction term in an effective action in the corotating frame of the star, and fix the coefficient by considering the spin-induced shift in the resonance frequencies that has been computed numerically for the mode frequencies of rotating neutron stars in the literature. We investigate the impact of relativistic corrections due to the gravitational redshift and frame-dragging effects, and identify important directions where more detailed theoretical developments are needed in the future. Comparisons of our model to numerical-relativity simulations of double neutron star and neutron star black-hole binaries show improved consistency in the agreement compared to current models used in data analysis.
\end{abstract}

DOI: 10.1103/PhysRevResearch.3.033129

\section{INTRODUCTION}

The gravitational waves (GWs) from inspiraling binary systems encode detailed information about the nature and internal structure of the compact objects. These signatures arise from spin and tidal effects, including dynamical tides associated with the excitation of the objects' characteristic quasinormal modes. This is particularly interesting for neutron stars (NSs), where gravity compresses matter up to several times the normal nuclear density [1,2], making NSs unique laboratories for the ground state of strongly interacting matter at the highest physically possible densities. The new opportunities for characterizing such matter with GWs were demonstrated with the first binary NS merger event GW170817 [3-23]. In the future, higher-precision GW measurements for populations of NS have the potential to advance our understanding of the fundamental physics of strong interactions as well as the emergent multibody phenomena in subatomic matter. Extracting the information on matter from

Published by the American Physical Society under the terms of the Creative Commons Attribution 4.0 International license. Further distribution of this work must maintain attribution to the author(s) and the published article's title, journal citation, and DOI. Open access publication funded by the Max Planck Society.
GW signals from binaries is critically predicated on highly accurate theoretical waveform models that link between features in GWs and source parameters [24-26]. In particular, the waveform models must include all relevant physical effects. This requires a detailed understanding of the behavior of matter in spinning, relativistic objects under nonlinear, dynamical gravity, which is a challenging task.

A significant research effort in the last decades has focused on developing GW models for binary black holes (BHs), which involve only vacuum gravity and are characterized by only their masses and spins [27-29]. There has also been much recent progress on describing the effects of matter in binary inspirals. A number of studies have focused on GW signatures of dynamical tides associated with different NS modes within different approximations [30-62], rotational multipole moments [63-73], gravitomagnetic tidal interactions [74-83], eccentricity [84-87], nonlinear mode couplings $[78,88,89]$, spin-tidal couplings in the adiabatic limit [72,90-98], and the effects of spins on the tidal response of black holes [99-102] as well as on dynamical tides in NSs in the Newtonian limit [103-109]. Recently, effectivefield-theory calculations of tidal effects in scattering events have also come into focus [110,111] (see also Ref. [112], and Refs. [113-116] for analogous work based on massive quantum fields or scattering amplitudes).

Substantial further effort has gone into developing state-of-the-art waveform models for data 
analysis within the so-called phenomenological IMRPhenom [117-131] and effective-one-body (EOB) SEOBNR/TEOBResumS families [132-164] (see also [165-171]). These models all include the effects of spin-induced multipole moments and the dominant tidal effects characterized by equation-of-state-dependent tidal deformability (or Love number) coefficients. In previous work, we calculated the effects of dynamical tides from the fundamental $(f)$ modes and incorporated them in the SEOBNR models [172,173], leading, e.g., to the SEOBNRv4T model. However, our model of dynamical tides had several limitations. For instance, we did not consider the effects of spin on the tidal response of the NS, which is the most prominent effect of spin-matter interactions.

In this paper, we extend the parameter space of waveform models by accounting for these spin effects in an approximate way, and investigate their role in NSNS and NSBH binaries. As expected on physical grounds and confirmed in previous work, e.g., [103,104,174], the effect of spins on dynamical $f$-mode tides can significantly enhance the matter signatures in GWs in the late inspiral for antialigned spins, depending also on the parameters. We show in this paper that this can lead to non-negligible dephasings with current data analysis models which neglect this effect. It is therefore urgent to model dynamical tides of rotating NS to enable the robustness of using GWs as probes for subatomic physics as the LIGO [175], Virgo [176], and KAGRA [177] GW detectors are improving in sensitivity and new, third-generation facilities are being envisioned. We study three main effects that influence the orbital frequency in a binary in which a rotating NS's $f$ mode is resonantly excited by the tidal field of the companion: (i) the gravitational redshift of the NS, (ii) the relativistic dragging of the NS's inertial frame, including also the additional effects of the orbiting companion, and (iii) the Coriolis effect due to the NS's spin. We derive an estimate for the resonant orbital frequency which approximately takes into account all of these effects and demonstrate that the most important effect is due to the NS's spin because of near cancellations between the redshift and frame-dragging effects. We develop a simple modification of the existing $f$-mode EOB waveform model which includes the Coriolis effect and is based on introducing spin-dependent shifts in the $f$-mode frequency and tidal deformability coefficients. We test our model against results from numerical-relativity simulations both for aligned and antialigned spins and find improved consistency compared to current models used in data analysis. Our simple model can readily be used to improve GW measurements. A more detailed theoretical study and model development which also overcomes other limitations and includes dynamical tides in the odd-parity sector will be the subject of forthcoming works.

The organization of this paper is as follows. We begin in Sec. II by deriving a Newtonian action for quadrupolar, parity-even dynamical tides in rotating stars. We start from a description in terms of the normal modes for the fluid displacement due to the perturbations and convert to a basis of symmetric trace-free tensors. That basis is more convenient for identifying selection rules, and for generalizing to relativistic stars. Such a generalization is worked out in Sec. III in the corotating frame where the background (unperturbed) fluid is at rest, provided we allow for general coupling coefficients not restricted to their Newtonian values. We specialize to the case of $f$ modes, which have the largest tidal couplings, and work to linear order in the rotation frequency. This leads to an effective action with one as yet undetermined coefficient characterizing the Coriolis interaction between the star's spin and its tidal spin, i.e., the angular momentum associated with the dynamical quadrupole. In Sec. IV we extend the action to a binary system and derive explicit equations of motion within the post-Newtonian approximation for the orbital dynamics. From the solutions for the quadrupole we obtain the response function whose features we analyze in Sec. V. We discuss how to determine the spin-tidal Coriolis coefficient by matching to results for the $f$-mode frequencies of rotating neutron stars from the literature and obtain a quasiuniversal relation for this shift. Next, we consider the impact of relativistic effects (gravitational redshift and frame dragging) on the dynamical tides and quantify their importance. In Sec. VI we derive a simple phenomenological model that accounts for the Coriolis effect by applying spin-dependent shifts of the $f$-mode frequency and tidal deformability parameter in the existing SEOBNRv4T waveform model. We test this model against numerical-relativity simulations of spinning binary neutron star and neutron star: black-hole binaries from the BAM and SXS codes in Sec. VII. Section VIII contains our conclusions, and the Appendix contains a brief discussion of the relation of this paper to Ref. [104].

The notation here follows that in Ref. [173]. We use geometric units with $G=c=1$ throughout. Capitalized indices $A, B, \ldots$ on tensors denote the representation in the spatial corotating frame and take values $1,2,3$. Greek letters $\mu$, $v, \ldots$ denote space-time coordinate indices and run through $0,1,2,3$. Lower-case indices $i, j, \ldots$ run through $1,2,3$ and denote either spatial coordinate indices when used on position variables or indices in a local Euclidean frame comoving with the center of the star for other tensors (spin, quadrupole) $[66,173]$. Boldface notation for vectors with such indices is also used. Round brackets around indices denote the symmetrization, square brackets denote the corresponding antisymmetric combination, and angle brackets denote symmetric trace-free projection. Our convention for the Riemann tensor is

$$
R^{\mu}{ }_{\nu \alpha \beta}=\Gamma_{\nu \beta, \alpha}^{\mu}-\Gamma_{\nu \alpha, \beta}^{\mu}+\Gamma_{\nu \beta}^{\rho} \Gamma_{\rho \alpha}^{\mu}-\Gamma_{\nu \alpha}^{\rho} \Gamma_{\rho \beta}^{\mu},
$$

where $\Gamma_{\nu \beta}^{\mu}$ is the Christoffel symbol. In the derivations we consider the case with only one extended body, which we label as body 1 with mass $m_{1}$. Since we work in the regime of linearized tides, the case of two stars can be obtained by adding the same contribution with the body labels exchanged. For a binary system, we define the total mass $M=m_{1}+m_{2}$, the reduced mass $\mu=m_{1} m_{2} / M$, and the symmetric mass ratio $v=\mu / M$.

\section{NEWTONIAN DYNAMICAL TIDES OF ROTATING STARS}

In this section we recapitulate Newtonian tides as linear perturbations of a background solution for a star in equilibrium following Refs. [42,49,83,173,178] (see also, e.g., Refs. $[46,179,180])$. The perturbation is described by a 
displacement vector field of the fluid elements $\boldsymbol{\xi}(\boldsymbol{x}, t)$ away from their background position. It is useful to consider the function space of all displacements as a complex Hilbert space with an inner product

$$
\left\langle\boldsymbol{\xi}, \boldsymbol{\xi}^{\prime}\right\rangle=\int d^{3} x \rho_{0} \boldsymbol{\xi}^{*} \cdot \boldsymbol{\xi}^{\prime},
$$

where $\rho_{0}$ is the unperturbed mass density of the background configuration.

We restrict the discussion here to an ideal fluid with barotropic equation of state $\rho(p)$ relating the mass density $\rho$ and the isotropic pressure $p$. That is, we neglect effects from, e.g., temperature, viscosity, and buoyancy, which play a subdominant role for the fundamental modes in neutron stars. We will first briefly recall the nonrotating case and obtain the Lagrangian describing the dynamics of the tidal perturbations, then generalize to include effects of spin to linear order in the rotation frequency, and finally transform to a description in terms of symmetric trace-free tensors.

\section{A. Nonrotating stars}

We first consider a nonrotating, hence spherically symmetric, star in equilibrium with density $\rho_{0}(r)$. The star is then placed in an external gravitational potential $\Phi$, which induces dynamical perturbations to the fluid. We will consider the perturbations only to linear order. For instance, the mass density perturbation is $\delta \rho=-\nabla\left(\rho_{0} \xi\right)$, where $\boldsymbol{\xi}=\boldsymbol{\xi}^{*}$ is the physical fluid displacement. The equations of motion for the dynamical tidal perturbations can be derived from a Lagrangian for the fluid displacement given by

$$
L_{\mathrm{DT}}=\frac{1}{2}\langle\dot{\boldsymbol{\xi}}, \dot{\boldsymbol{\xi}}\rangle-\frac{1}{2}\langle\boldsymbol{\xi}, \mathcal{D} \boldsymbol{\xi}\rangle+\left\langle\boldsymbol{f}^{\mathrm{ext}}, \boldsymbol{\xi}\right\rangle .
$$

The first term in Eq. (2.2) is the kinetic energy of the perturbation, the second term specifies the energies associated with the internal restoring forces, while the last term is the potential energy in the external field. For the case considered in this paper, the external force ${ }^{1}$ is $f^{\text {ext }}=-\nabla \Phi$ where $\Phi$ is the gravitational potential of a binary companion orbiting at a distance $\boldsymbol{r}(t)$ and given by

$$
\Phi=-\frac{m_{2}}{|\boldsymbol{x}-\boldsymbol{r}(t)|} .
$$

The linear operator $\mathcal{D}$ is defined by

$$
\mathcal{D} \boldsymbol{\xi}=-\nabla\left\{\left[\frac{c_{s}^{2}}{\rho_{0}}+4 \pi \Delta^{-1}\right] \nabla \cdot\left(\rho_{0} \xi\right)\right\},
$$

where $c_{s}=\sqrt{\left(\partial p_{0} / \partial \rho_{0}\right)}$ is the speed of sound of the background fluid configuration with pressure $p_{0}$. The first term in Eq. (2.4) comes from the perturbation of the internal energy, and the second (nonlocal) term describes the gravitational self-energy of the perturbation.

The operator $\mathcal{D}$ is Hermitian with respect to the inner product (2.1). Thus, its eigenvectors $\boldsymbol{\xi}_{n \ell m}$ are an orthonormal basis of the normal modes labeled by the type of mode $n$,

\footnotetext{
${ }^{1}$ We disregard here the fictitious force arising from the center-ofmass acceleration of the star (see, e.g., [173]), which effectively just cancels $f^{\text {ext }}$ in the dipolar sector (equivalence principle).
}

the multipolar order $\ell$, and an angular-momentum number $m$ associated with a decomposition into (vector) spherical harmonics. The real eigenvalues $\omega_{n \ell}^{2}$, where $\omega_{n \ell}$ is the mode frequency, are determined from

$$
\mathcal{D} \boldsymbol{\xi}_{n \ell m}=\omega_{n \ell}^{2} \boldsymbol{\xi}_{n \ell m}, \quad\left\langle\boldsymbol{\xi}_{n \ell m}, \boldsymbol{\xi}_{n^{\prime} \ell^{\prime} m^{\prime}}\right\rangle=\delta_{n n^{\prime}} \delta_{\ell \ell^{\prime}} \delta_{m m^{\prime}} .
$$

The mode frequencies $\omega_{n \ell}$ are degenerate over $m$ because the operator $\mathcal{D}$ is rotation symmetric. Similarly, due to parity invariance of $\mathcal{D}$, the modes can be categorized as even parity (electric type) or odd parity (magnetic type). As the integration measure $\rho_{0} d^{3} x$ of the inner product (2.1) has compact support, the normal modes $\boldsymbol{\xi}_{n \ell m}$ are countable and are enumerated by the number $n$ (aside from $\ell$ and $m$ ). We restrict our attention to pressure modes in this paper and take $n$ to be the number of radial nodes. The fundamental pressure mode or $f$ mode is then labeled by $n=0$.

We can decompose any fluid displacement $\boldsymbol{\xi}$ into the orthonormal basis of the normal modes,

$$
\boldsymbol{\xi}=\sum_{n \ell m} q_{n \ell m}(t) \boldsymbol{\xi}_{n \ell m}(\boldsymbol{x}), \quad q_{n \ell m}=\left\langle\boldsymbol{\xi}_{n \ell m}, \boldsymbol{\xi}\right\rangle,
$$

with time-dependent amplitudes $q_{n \ell m}(t)$. The reality condition $\xi=\xi^{*}$ implies that $q_{n \ell m}^{*}=(-1)^{m} q_{n \ell-m}$, which follows from the analogous relation for the spherical harmonics. The general Lagrangian then reads as

$$
L_{\mathrm{DT}}=\sum_{n \ell m}\left[\frac{1}{2}\left|\dot{q}_{n \ell m}\right|^{2}-\frac{1}{2} \omega_{n \ell}^{2}\left|q_{n \ell m}\right|^{2}+\left\langle f^{\mathrm{ext}}, \xi_{n \ell m}\right\rangle q_{n \ell m}\right] .
$$

To compute the coefficients for the overlap between the external field and the mode functions, and to identify the modes giving the most important contributions to $L_{\mathrm{DT}}$, it is useful to express the potential from Eq. (2.3) as a Taylor series expansion around the center of the star. Choosing coordinates such that the center of the star is located at $\boldsymbol{x}=0$, the expansion of the potential is

$$
\Phi(t, \boldsymbol{x})=\Phi(t, \mathbf{0})+\left.x^{j} \partial_{j} \Phi(t, \boldsymbol{x})\right|_{\boldsymbol{x}=0}+\sum_{\ell=2}^{\infty} \frac{1}{\ell !} x^{L} E_{L},
$$

where the $\ell$ th tidal moments $E_{L}$ for $\ell \geqslant 2$ are defined by (following the conventions in [173])

$$
E_{L}=\left.\partial_{L} \Phi\right|_{x=0}
$$

and $L=A, B, C, \ldots$ denotes a string of $\ell$ indices. Note that $E_{L}$ is symmetric and trace free, which follows from $\left.\Delta \Phi\right|_{x=0}=0$. The tidal potential can equivalently be written as a spherical harmonic multipolar expansion given by

$$
\Phi=-m_{2} \sum_{\ell, m} \frac{4 \pi}{2 \ell+1} \frac{|\boldsymbol{x}|^{\ell}}{r(t)^{\ell+1}} Y_{\ell m}\left(\frac{\pi}{2}, \phi\right) Y_{\ell m}^{*}(\theta, \varphi),
$$

where $\boldsymbol{x}$ and $(\theta, \varphi)$ are associated with a comoving coordinate system centered on the star, and $r, \phi$ characterize the orbital coordinates in the equatorial plane. We define the overlap integral $I_{n \ell}$ by

$$
I_{n \ell}=\left\langle\nabla|\boldsymbol{x}|^{\ell} Y_{\ell m}(\theta, \varphi), \boldsymbol{\xi}_{n \ell m}\right\rangle .
$$

The term $\left\langle\boldsymbol{f}^{\text {ext }}, \boldsymbol{\xi}_{n \ell m}\right\rangle$ in the Lagrangian can then be written as

$$
\left\langle\boldsymbol{f}^{\mathrm{ext}}, \boldsymbol{\xi}_{n \ell m}\right\rangle=-\frac{N_{\ell}}{\ell !} I_{n \ell} E_{\ell m}^{*},
$$


where

$$
E_{\ell m}=N_{\ell} \mathcal{Y}_{L}^{* \ell m} E_{L}=-(2 \ell-1) ! ! N_{\ell} Y_{\ell m}^{*}\left(\frac{\pi}{2}, \phi\right) \frac{m_{2}}{r^{\ell+1}} .
$$

This can be either obtained from Eq. (2.9) or its sphericalharmonic analog (2.10). Recall that one can convert between spherical harmonics and unit vectors using the identity $Y_{\ell m}=$ $\mathcal{Y}_{L}^{\ell m} n^{\langle L\rangle}$ and defining the coefficient $N_{\ell}=\sqrt{4 \pi \ell ! /(2 \ell+1) ! !}$ that arises when applying the inverse conversion to change from $n^{\langle A} n^{B\rangle}$ to $Y_{\ell m}$; see, e.g., Ref. [83] for useful formulas.

The modes with the largest contributions to $L_{\mathrm{DT}}$ can be identified by the following considerations. First, we note that the $\ell$ th multipole of the external tidal field associated with $\ell \geqslant$ 2 derivatives of $\Phi$ is increasingly suppressed for increasing multipole orders $\ell$. We thus expect the dominant contributions to come from the low- $\ell$ modes. However, the $\ell=0,1$, as well as all magnetic-type modes do not couple linearly to the external gravitational field in the Newtonian case. The $\ell=0$ interaction is forbidden due to the conservation of mass, while the $\ell=1$ interaction leads to an overall motion of the star, which has no gauge-invariant physical meaning according to the weak equivalence principle (universality of free fall). The magnetic modes couple linearly to gravitomagnetic tidal fields which is a relativistic phenomenon that is absent in Newtonian gravity. Hence, to leading order, the external field drives the electric quadrupolar $(\ell=2)$ modes, so we restrict our attention to them in the following.

\section{Transformation to the basis of symmetric trace-free Cartesian tensors}

We can equivalently express the Lagrangian in terms of symmetric trace-free tensors using the conversion between spherical harmonics and unit vectors provided by the symmetric trace-free tensors $\mathcal{Y}_{L}^{\ell m}$. The mode amplitudes $q_{n \ell m}$ can then be directly translated to Cartesian tensors. For the quadrupole $\ell=2$, we adopt the normalization

$$
Q_{n}^{A B}=\sqrt{2 \lambda_{n}} \omega_{n} N_{2} \sum_{m} \mathcal{Y}_{A B}^{2 m} q_{n 2 m},
$$

where $N_{2}=\sqrt{8 \pi / 15}, \omega_{n}=\omega_{n 2}$ is the mode frequency, and $\lambda_{n}$ is the tidal deformability of the mode, related here to the overlap integral by ${ }^{2}$

$$
\lambda_{n}=\frac{N_{2}^{2} I_{n 2}^{2}}{2 \omega_{n}^{2}}=\frac{4 \pi I_{n 2}^{2}}{15 \omega_{n}^{2}} .
$$

The total quadrupole is given by summing over all overtones

$$
Q_{A B}=\sum_{n=0}^{\infty} Q_{n}^{A B}
$$

We also define the Newtonian quadrupolar tidal tensor

$$
E^{A B}=\left.\partial_{A} \partial_{B} \Phi(\boldsymbol{x})\right|_{\boldsymbol{x}=0} .
$$

\footnotetext{
${ }^{2}$ Our convention for $I_{n \ell}$ differs from Refs. [49,173] by a factor of $N_{\ell}$ [see Eq. (2.2) in Ref. [173]].
}

The Lagrangian (2.7) can then be written as

$$
L_{\mathrm{DT}}=\sum_{n}\left[\frac{1}{4 \lambda_{n} \omega_{n}^{2}}\left(\dot{Q}_{n}^{A B} \dot{Q}_{n}^{A B}-\omega_{n}^{2} Q_{n}^{A B} Q_{n}^{A B}\right)-\frac{1}{2} E^{A B} Q_{n}^{A B}\right]
$$

We remind the reader that $Q_{n}^{A B}$ is the contribution of the $n$ mode to the (symmetric trace-free) quadrupole of the star, and $E^{A B}$ is the external tidal field evaluated at the center of the star. A key point to note is that because we work in a threedimensional rest frame of the star labeled by $A, B=1,2,3$ (or the corotating frame below), the structure of the couplings for the internal dynamics of the quadrupole is the same for Newtonian and relativistic stars [cf. Eq. (1.4) in [173]]; the distinction between them is only through the coefficients $\left(\lambda_{n}\right.$, $\left.\omega_{n}\right)$. We will exploit this fact for rotating stars below since we are interested here in fully relativistic NSs, where $\omega_{n}$ and $\lambda_{n}$ are computed in general relativity.

\section{B. Rotating stars}

It is straightforward to extend the discussion from the last section to stars that are rotating uniformly with an angular velocity of the star $\boldsymbol{\Omega}$ as observed in the inertial frame. It is convenient to describe the star in the corotating frame, where the background fluid elements are at rest. At linear order in $\boldsymbol{\Omega}$, the only new interaction with dynamical multipoles is due to the Coriolis force

$$
L_{\mathrm{DT}}=\frac{1}{2}\langle\dot{\boldsymbol{\xi}}, \dot{\boldsymbol{\xi}}\rangle-\langle\boldsymbol{\xi}, \boldsymbol{\Omega} \times \dot{\boldsymbol{\xi}}\rangle-\frac{1}{2}\langle\boldsymbol{\xi}, \mathcal{D} \boldsymbol{\xi}\rangle+\left\langle\boldsymbol{f}^{\mathrm{ext}}, \boldsymbol{\xi}\right\rangle
$$

The background star gets deformed away from spherical symmetry only at quadratic order in $\boldsymbol{\Omega}$, so that the eigenvectors and eigenvalues of $\mathcal{D}$ are approximately the same as for a spherically symmetric nonrotating star. Since we treat the spin as (infinitesimally) small, we decompose the displacement in terms of the nonrotating modes (2.6) and, working to linear order in spin, the perturbing interaction is given by the Coriolis force only. A detailed discussion of the more general finite-spin case can be found in Ref. [179].

Inserting the decomposition for $\boldsymbol{\xi}$ [Eq. (2.6)] leads to

$$
\begin{aligned}
L_{\mathrm{DT}}= & \sum_{n \ell m}\left[\frac{1}{2}\left|\dot{q}_{n \ell m}\right|^{2}-\frac{1}{2} \omega_{n \ell}^{2}\left|q_{n \ell m}\right|^{2}+\frac{1}{\ell !} I_{n \ell} E_{\ell m} q_{n \ell m}\right. \\
& \left.-\sum_{n^{\prime} \ell^{\prime} m^{\prime}} q_{n \ell m}^{*} \dot{q}_{n^{\prime} \ell^{\prime} m^{\prime}}\left\langle\boldsymbol{\xi}_{n \ell m}, \boldsymbol{\Omega} \times \boldsymbol{\xi}_{n^{\prime} \ell^{\prime} m^{\prime}}\right\rangle\right]
\end{aligned}
$$

The last term here represents a linear mode coupling (quadratic in the action) due to the rotation. These mode couplings are subject to selection rules. The selection rules are most readily identified in the symmetric trace-free basis where they are automatically implemented when imposing symmetry requirements. Specifically, the allowed couplings are all parity-invariant contractions between the symmetric trace-free tensors of the modes with either the parity-odd angular velocity vector $\boldsymbol{\Omega}$ or its associated antisymmetric parity-even tensor

$$
\Omega^{A B}=\epsilon_{A B C} \Omega^{C} .
$$


We focus here on the electric quadrupolar $(\ell=2)$ modes $Q_{n}^{A B}$. All possible spin-mode couplings involving $Q_{n}^{A B}$ read as

$$
Q_{n}^{A B} \dot{Q}_{n^{\prime}}^{B C} \Omega^{A C}, \quad Q_{n}^{A B} \dot{Q}_{n^{\prime}}^{B} \Omega^{A}, \quad Q_{n}^{A B} \dot{Q}_{n^{\prime}}^{A B C} \Omega^{C} .
$$

Hence, couplings to magnetic modes are possible to a dipole $Q_{n^{\prime}}^{B}$ and to an octupole $Q_{n^{\prime}}^{A B C}$, which we neglect since these magnetic modes are not externally driven in the Newtonian limit. We then arrive at the Lagrangian

$$
L_{\mathrm{DT}} \approx \sum_{n}\left[L_{o}-\frac{1}{2} E^{A B} Q_{n}^{A B}+L_{S Q}\right],
$$

where the oscillator and spin-mode contributions are given by

$$
\begin{gathered}
L_{o}=\frac{1}{4 \lambda_{n} \omega_{n}^{2}}\left(\dot{Q}_{n}^{A B} \dot{Q}_{n}^{A B}-\omega_{n}^{2} Q_{n}^{A B} Q_{n}^{A B}\right), \\
L_{S Q}=\sum_{n^{\prime}} C_{\Omega n n^{\prime}} \Omega^{A B} Q_{n}^{A C} \dot{Q}_{n^{\prime}}^{C B} .
\end{gathered}
$$

The effect of rotation is explicit here via the term $L_{\mathrm{SQ}}$ describing a rotation-induced coupling between different modes of the same multipolar order due to the Coriolis force. We have inserted the as-yet-undetermined coefficients $C_{\Omega n n^{\prime}}$. It is possible to write an explicit formula for $C_{\Omega n n^{\prime}}$, analogous to Eq. (2.11) for $I_{n \ell}$, that is valid in Newtonian gravity. However, we do not need this here since we are ultimately interested in the fully relativistic value of this coefficient. We will focus here on the fundamental $f$ modes with $n=n^{\prime}=0$ and determine all coefficients $\lambda_{0}, \omega_{0}, C_{\Omega 00}$ by matching to relativistic results for the effect of spin on the mode frequencies in Sec. VB below. We will drop the label $n$ on $Q$ from now on.

\section{RELATIVISTIC DYNAMICAL TIDES OF ROTATING STARS}

In this section, we upgrade the Newtonian action to a relativistic one, following the nonrotating case in Ref. [173]. For the treatment of the star's spin or angular momentum, we draw from Refs. [66,94,181-183]. The resulting action is an effective one, where length scales below the bodies' size are integrated out, and could also be constructed from an effective-field-theory approach [184-189]. However, we do not attempt here a rigorous construction of such an action based on symmetries and power-counting arguments. Instead, we only include terms in the relativistic action that are already present in the Newtonian case above, but with undetermined coefficients. We expect terms that are absent in the Newtonian limit to be suppressed for relativistic electric tides, which is also justified from numerical studies of the relativistic tidal response [190]. We note, however, that in the case of magnetic tides, such an approach would crucially miss important terms in the relativistic effective action, as discussed in [83]. The explicit results of calculations in the post-Newtonian approximation for the binary dynamics based on effective actions can be found, e.g., in Refs. [191-194].

\section{A. Upgrading the Newtonian action}

A relativistic rotating star can be represented by a world line $y^{\mu}(\tau)$ with dynamical tidal and spin degrees of freedom propagating along it. Here $\tau$ is the proper time and the tangent 4-velocity is given by $U^{\mu}=d y^{\mu}(\tau) / d \tau$ such that $U_{\mu} U^{\mu}=-1$. The rotation of the star can be encoded by an orthonormal corotating, body-fixed frame $\Lambda_{I}{ }^{\mu}(\tau)$ on the world line (with $I, J, \cdots=1,2,3$ and $\Lambda_{I}{ }^{\mu} \Lambda_{J \mu}=\delta_{I J}$ ) describing the orientation of the star. This frame is taken to be comoving such that $\Lambda_{I}{ }^{\mu} U_{\mu}=0$. Based on this frame, we can define the relativistic angular velocity in the corotating frame as

$$
\Omega^{I}=\epsilon^{I J K} \Omega_{J K}, \quad \Omega_{J K}=\frac{D \Lambda_{I}^{\mu}}{d \tau} \Lambda_{J \mu}
$$

where $D$ is the covariant differential. The external tidal field is given by the electric part of the Weyl tensor as $E_{I J}=$ $\Lambda_{I}{ }^{\mu} U^{\alpha} \Lambda_{J}{ }^{\nu} U^{\beta} C_{\mu \alpha \nu \beta}$ in the relativistic case, where $C_{\mu \alpha \nu \beta}$ is the Weyl curvature tensor. The relativistic $f$-mode amplitude $Q^{A B}(\tau) \equiv Q_{0}^{A B}(\tau)$ is written in the corotating frame along the world line. Time derivatives are taken with respect to proper time and denoted by an overdot ${ }^{\circ}=d / d \tau$.

It is now straightforward to upgrade the above Newtonian Lagrangian (2.23) to the relativistic case: the structure remains the same but all the quantities must be computed from the appropriate relativistic definitions discussed above. For the description of a binary, one needs to supplement the action $\mathcal{S}$ by a nontidal (NT) part,

$$
\begin{aligned}
\mathcal{S} & =\int d \tau(\underbrace{L_{\mathrm{NT}}+L_{\mathrm{DT}}}_{L}), \\
L_{\mathrm{NT}} & =-m_{0}+\frac{I}{2} \Omega_{I} \Omega^{I}+\cdots,
\end{aligned}
$$

with the irreducible (rotation-independent) mass $m_{0}$, the moment of inertia $I$, and the dots representing further terms not relevant here (e.g., from the spin-induced quadrupole moment [195-197]).

\section{B. Legendre transformation}

The spin-tidal interaction due to the Coriolis force specialized to $n=n^{\prime}=0$ can also be expressed as a coupling between the star's spin and the tidal spin associated with the $f$ modes as we will show next. This highlights the connection to spin or frame-dragging effects in general relativity. Following Ref. [173], we define a tidal spin tensor and vector associated with the quadrupolar $f$ modes by

$$
S_{Q}^{A}=\frac{1}{2} \epsilon_{B C}^{A} S_{Q}^{B C}, \quad S_{Q}^{A B}=4 Q^{C[A} P^{B]}{ }_{C},
$$

where

$$
P_{A B}=\frac{\partial L}{\partial \dot{Q}^{A B}}
$$

is the conjugate momentum to $\dot{Q}_{A B}$. We also introduce the total spin $S_{t}^{I}$ conjugate to the rotation frequency

$$
S_{t}^{I}=\frac{\partial L}{\partial \Omega_{I}},
$$

and the associated spin tensor $S_{t}^{I J}=\epsilon^{I J}{ }_{K} S_{t}^{K}$. To zeroth order in the tidal contributions $S_{t}^{J} \approx I \Omega^{J}$. 
We perform a Legendre transformation of the Lagrangian to $P_{A B}$ and $S_{t}^{A}$, which leads to the action

$$
\begin{gathered}
\mathcal{S}=\int d \tau\left(S_{t}^{A} \Omega_{A}+P_{A B} \dot{Q}^{A B}+R\right), \\
R \approx-m_{0}-\frac{1}{2 I} S_{t A} S_{t}^{A}+C_{S Q} S_{t A} S_{Q}^{A}-\frac{1}{2} E_{A B} Q^{A B} \\
-\frac{1}{4 \lambda_{0} \omega_{0}^{2}}\left(4 \lambda_{0}^{2} \omega_{0}^{4} P_{A B} P^{A B}+\omega_{0}^{2} Q_{A B} Q^{A B}\right),
\end{gathered}
$$

where we neglected terms beyond quadratic order in the tidal variables and defined

$$
C_{S Q}=C_{\Omega 00} \frac{\lambda_{0} \omega_{0}^{2}}{I} .
$$

We see that the leading-order spin-tidal interaction can be understood as a spin-spin interaction between the ordinary and tidal spins $S_{t A} S_{Q}^{A}$. We note that due to the dynamical tidal interactions, the spin length $S_{t}=\sqrt{S_{t A} S_{t}^{A}}$ is not constant.

\section{Coordinate-frame action}

Let us now connect the relativistic action (3.7) to that of the nonspinning case discussed in Ref. [173], which is formulated in the coordinate frame instead of the corotating frame. The transformation of the above results to the coordinate frame is simply given by $Q^{A B}=\Lambda^{A \mu} \Lambda^{B \nu} Q_{\mu \nu}$, and similarly for the other tensors. This leads to the relation

$$
P_{A B} \dot{Q}^{A B}=P_{\mu \nu} \frac{D Q^{\mu \nu}}{d \tau}-\Omega_{\mu} S_{Q}^{\mu} .
$$

It is convenient to absorb the second term by splitting the total spin $S_{t}^{\mu}$ into a "rotational-only" spin $S^{\mu}$ and the tidal part $S_{Q}^{\mu}$ :

$$
S_{t}^{\mu}=S^{\mu}+S_{Q}^{\mu} .
$$

The coordinate-frame action then reads as

$$
\begin{gathered}
\mathcal{S}=\int d \tau\left[S^{\mu} \Omega_{\mu}+P_{\mu \nu} \frac{D Q^{\mu \nu}}{d \tau}+R\right], \\
R \approx-m\left(S^{2}\right)+\bar{C}_{S Q} S_{\mu} S_{Q}^{\mu}-\frac{1}{2} E_{\mu \nu} Q^{\mu \nu} \\
-\frac{1}{4 \lambda_{0} \omega_{0}^{2}}\left(4 \lambda_{0}^{2} \omega_{0}^{4} P_{\mu \nu} P^{\mu \nu}+\omega_{0}^{2} Q_{\mu \nu} Q^{\mu \nu}\right),
\end{gathered}
$$

where the coordinate-frame coefficient associated with the spin-tidal interaction is given by

$$
\bar{C}_{S Q}=C_{S Q}-\frac{1}{I} .
$$

We are going to find below that this equation just encodes the difference of the $f$-mode frequency between corotating and inertial frames. We have introduced the constant ArnowittDeser-Misner mass (not to be confused with the magnetic number $m$ )

$$
m\left(S^{2}\right)=m_{0}+\frac{1}{2 I} S^{2}+O\left(S^{4}\right) .
$$

Note that the spin length is now constant, $S^{2}=S_{\mu} S^{\mu}=$ const. Furthermore, since $\Lambda_{I}^{\mu} U_{\mu}=0$, all coordinate-frame tensors are orthogonal to the 4-velocity:

$$
\begin{aligned}
& \Omega_{\mu} U^{\mu}=0, \quad Q^{\mu \nu} U_{\nu}=0, \\
& S^{\mu} U_{\mu}=0, \quad P_{\mu \nu} U^{\nu}=0 .
\end{aligned}
$$

These constraints have to be fulfilled alongside the variational principle for the action.

\section{POST-NEWTONIAN APPROXIMATION}

The action above (3.12) models a single neutron star interacting with an external gravitational field as a worldline (point-particle) effective action with spin and dynamical quadrupole moment. Based on this building block, the action of a binary system can be constructed as two copies of Eq. (3.12) together with the Einstein-Hilbert action for the gravitational field. One can further eliminate (integrate out) the orbital-scale field within the post-Newtonian approximation, which is a weak-field and slow-motion approximation around the Newtonian limit. This can be understood as a formal expansion in the inverse speed of light. We discuss this post-Newtonian approximate action for a binary system in this section.

\section{A. Post-Newtonian action and Hamiltonian}

Instead of performing the post-Newtonian calculation in detail, we can take a shortcut by building on previous results. Indeed, the world-line action (3.12) is a sum of the nonspinning dynamical tidal action from Ref. [173] and the spin action from, e.g., Ref. [66], plus the simple spin-tidal correction $\bar{C}_{S Q} S_{\mu} S_{Q}^{\mu}$. Since we work to linear order in spin and tidal interactions, the result for the Lagrangian of a binary system in the post-Newtonian approximation can be taken from these references and by adding the spin-tidal interaction for each body. This leads to

$$
\mathcal{S}_{\mathrm{SQ}}=\int d \tau \bar{C}_{S Q} \boldsymbol{S}_{Q} \cdot \boldsymbol{S}=\int d t z \bar{C}_{S Q} \boldsymbol{S}_{Q} \cdot \boldsymbol{S},
$$

where $z=d \tau / d t=\sqrt{g_{\mu \nu} \dot{y}^{\mu} \dot{y}^{\nu}}$ is the redshift variable. In this section, the meaning of an overdot changes compared to Sec. III and now denotes a derivative with respect to coordinate time $=d / d t$. In the post-Newtonian results, the temporal components of spin and tidal variables are eliminated by writing them in a comoving local Euclidean frame and using Eq. (3.16) (see Refs. [66,173] for details). The spatial components in this frame are simply denoted as, e.g., $Q^{i j}$ where $i, j=1,2,3$.

We assume that only one of the objects has a finite size and label it as body 1 . Within our approximations, the case of two extended objects can readily be obtained by adding the same tidal contributions with the appropriate parameters for the other object. The action for the binary in the center-ofmass frame in Hamiltonian form has the structure

$$
\mathcal{S}_{\mathrm{PN}}=\int d t\left(\boldsymbol{p} \cdot \boldsymbol{r}+\boldsymbol{S}_{1} \cdot \boldsymbol{\Omega}_{1}+\boldsymbol{S}_{2} \cdot \boldsymbol{\Omega}_{2}+P^{i j} \dot{Q}^{i j}-H_{\mathrm{PN}}\right) .
$$

Here, $\boldsymbol{p}$ is the relative linear momentum and $\boldsymbol{r}$ is the separation vector. The post-Newtonian Hamiltonian splits as $H_{\mathrm{PN}}=$ $H_{\mathrm{NT}}+H_{\mathrm{DT}}$ into a nontidal $H_{\mathrm{NT}}$ and a dynamical-tidal $H_{\mathrm{DT}}$ 
part with [173]

$$
H_{\mathrm{DT}}=z H_{o}+\boldsymbol{\Omega}_{\mathrm{FD}} \cdot \boldsymbol{S}_{Q}-z \bar{C}_{S Q} \boldsymbol{S}_{1} \cdot \boldsymbol{S}_{Q}+\frac{z}{2} E_{i j} Q^{i j} .
$$

Different versions of the nontidal Hamiltonian $H_{\mathrm{NT}}=$ $H_{\mathrm{NT}}\left(\boldsymbol{r}, \boldsymbol{p}, \boldsymbol{S}_{1}, \boldsymbol{S}_{2} ; m_{1}, m_{2}, C_{W}\right)$ exist in the literature, e.g., in Refs. [198,199]; the precise version will not be important here. Here $C_{W}$ collectively denotes several Wilson coefficients in the original world-line effective action that describes, e.g., spin-induced multipole moments. Furthermore, the redshift $z$ and frame-dragging and spin-precession frequency $\boldsymbol{\Omega}_{\mathrm{FD}}$ in the Hamiltonian (4.3) are given by

$$
z=\frac{\partial H_{\mathrm{NT}}}{\partial m_{1}}, \quad \boldsymbol{\Omega}_{\mathrm{FD}}=\left.\frac{\partial H_{\mathrm{NT}}}{\partial \boldsymbol{S}_{1}}\right|_{C_{W}=0},
$$

and the oscillator-part Hamiltonian reads as

$$
H_{o}=\frac{1}{4 \lambda_{0} \omega_{0}^{2}}\left(4 \lambda_{0}^{2} \omega_{0}^{4} P^{i j} P^{i j}+\omega_{0}^{2} Q^{i j} Q^{i j}\right) .
$$

Finally, the post-Newtonian tidal field $E_{i j}=E_{i j}\left(\boldsymbol{r}, \boldsymbol{p} ; m_{1}, m_{2}\right)$ in the comoving local Euclidean frame [173] can be found in Refs. $[173,191]$ in different gauges. To leading Newtonian order, the tidal field follows from Eq. (2.9):

$$
E_{i j}=-\frac{3 m_{2}}{r^{3}} n^{\langle i} n^{j\rangle}+\cdots,
$$

where $r=|\boldsymbol{r}|$ and $\boldsymbol{n}=\boldsymbol{r} / r$; the explicit form of higher-order corrections will be irrelevant for our studies below.

\section{B. Circular-orbit tidal equations of motion}

From now on, we assume that the binary is on a circular orbit and that the spins are not precessing, i.e., they are aligned or antialigned with the orbital angular momentum. For generic orbits, different gauge choices for $H_{\mathrm{NT}}$ lead to different expressions for $z$ and $\boldsymbol{\Omega}_{\mathrm{FD}}$. For circular orbits and nonprecessing spins, however, they are universally given by

$$
\begin{aligned}
z= & 1+\frac{x}{2}\left(v-3 X_{2}\right) \\
& +\frac{x^{2}}{24}\left(5 v^{2}-9 v-6 v X_{2}-27 X_{2}\right)+O\left(x^{5 / 2}\right), \\
\frac{\Omega_{\mathrm{FD}}}{\omega_{\mathrm{orb}}}= & \frac{x}{2}\left(v+3 X_{2}\right)-x^{3 / 2} X_{2}^{2} \chi_{2} \\
& -\frac{x^{2}}{24}\left(v^{2}-45 v+30 v X_{2}-27 X_{2}\right)+O\left(x^{5 / 2}\right),
\end{aligned}
$$

where $\Omega_{\mathrm{FD}}=\left|\boldsymbol{\Omega}_{\mathrm{FD}}\right|$. We have also defined the spin magnitude $\chi_{2}= \pm\left|\boldsymbol{S}_{2}\right| / m_{2}^{2}$ and the mass ratio $X_{2}=m_{2} / M$ for the companion, and introduced the frequency variable $x=\left(M \omega_{\text {orb }}\right)^{2 / 3}$ where $\omega_{\text {orb }}$ is the orbital frequency. For aligned companion spin, $\chi_{2}>0$, while for antialigned spin it is $\chi_{2}<0$. Here the power on $x$ corresponds to the post-Newtonian order. Note that for circular orbits, the binary is in equilibrium, so that $\omega_{\mathrm{orb}}, z$, and $\Omega_{\mathrm{FD}}$ are constant.

An interesting observation here is that the frame dragging due to the orbital angular momentum given by the first term in (4.8) and that resulting from the companion spin, given by the second term in (4.8), have opposite signs. This can be understood by visualizing the directed gravitomagnetic field lines analogous to a bar magnet (see, e.g., the discussion in
Ref. [200]). The neutron star experiences the gravitomagnetic field from the orbital motion at its source ("inside the gravitomagnet"), where the field lines point in the same direction as the orbital angular momentum. Conversely, the field of the companion felt by the star is outside its source, where the gravitomagnetic field lines point in the opposite direction of $\boldsymbol{S}_{2}$. This implies that for aligned companion spins, the net frame-dragging effects are smaller than for antialigned spins of the companion.

Hamilton's equations of motion follow from varying the tidal variables in the action (4.2),

$$
\dot{Q}^{i j}=\frac{\partial H_{\mathrm{DT}}}{\partial P^{i j}}, \quad \dot{P}^{i j}=-\frac{\partial H_{\mathrm{DT}}}{\partial Q^{i j}}
$$

or, more explicitly,

$$
\begin{aligned}
& \dot{Q}^{i j}=2 z \lambda_{0} \omega_{0}^{2} P^{i j}+2 \Omega_{\mathrm{FD}}^{k(i} Q^{j) k}-2 z \bar{C}_{S Q} S_{1}^{k(i} Q^{j) k} \\
& \dot{P}^{i j}=-\frac{z}{2 \lambda_{0}} Q^{i j}+2 \Omega_{\mathrm{FD}}^{k(i} P^{j) k}-2 z \bar{C}_{S Q} S_{1}^{k(i} P^{j) k}-\frac{z}{2} E_{i j} .
\end{aligned}
$$

These equations can be decoupled by transforming to the spherical-harmonic $(\ell, m)$ basis. For this purpose, we express the relativistic quadrupole due to the $f$ modes as $Q^{i j}=$ $N_{2} \sum_{k} \mathcal{Y}_{A B}^{2 m} Q_{m}$, and similar for $P^{i j}$ and $E_{i j}$, analogous to the transformation in the Newtonian case discussed in Sec. II A. The reality condition implies that $Q_{m}^{*}=(-1)^{m} Q_{-m}$. Furthermore, we choose to align the $z$ axis with the spin so that $\left(S_{1}^{i}\right)=S_{1}(0,0,1)$. Since we also assume that the spins are collinear with the orbital angular momentum, this means that $\boldsymbol{\Omega}_{\mathrm{FD}}$ is along the $z$ axis as well. This leads us to the equations of motion

$$
\begin{gathered}
\dot{Q}_{m}=2 z \lambda_{0} \omega_{0}^{2} P_{m}-i m\left(\Omega_{\mathrm{FD}}-z \bar{C}_{S Q} S_{1}\right) Q_{m}, \\
\dot{P}_{m}=-\frac{z}{2 \lambda_{0}} Q_{m}-i m\left(\Omega_{\mathrm{FD}}-z \bar{C}_{S Q} S_{1}\right) P_{m}-\frac{z}{2} E_{m} .
\end{gathered}
$$

These equations can be combined into a single second-order differential equation describing the $f$-mode oscillations

$$
\left[\partial_{t}+i m\left(\Omega_{\mathrm{FD}}-z \bar{C}_{S Q} S_{1}\right)\right]^{2} Q_{m}+z^{2} \omega_{0}^{2} Q_{m}=-z^{2} \omega_{0}^{2} \lambda_{0} E_{m} .
$$

For the driving force at Newtonian order it holds $E_{m}=$ $\mathcal{E}_{m} e^{-i m \omega_{\text {orb }} t}$ with

$$
\mathcal{E}_{ \pm 2}=-\sqrt{\frac{3}{2}} \mathcal{E}_{0}=-\frac{3 m_{2}}{2 M^{3}} x^{3}, \quad \mathcal{E}_{ \pm 1}=0 .
$$

Note that in the Newtonian limit $x \approx M / r$. This can be obtained from Eq. (2.13) noting that $E_{m}=E_{2 m}$ and $\phi=\omega_{\text {orb }} t$. The latter relation is consistent with our assumption of an equilibrium solution, but only holds approximately for an inspiral and breaks down close to the resonance, where $\omega_{\text {orb }}$ changes in time. This will be discussed further below in connection with the effective Love number.

\section{EXPLORING THE TIDAL RESPONSE}

In this section, we derive the frequency-domain response of the quadrupole $Q^{i j}$ to the tidal field $E_{i j}$ in a binary system described by the post-Newtonian Hamiltonian $H_{\mathrm{DT}}$. We emphasize again that we are treating $Q^{i j}$ as fully relativistic, and 
only use the post-Newtonian approximation for quantities related to the orbital dynamics. We assume again a circular-orbit nonprecessing binary. Our goal is to investigate the impact of the redshift, frame-dragging, and spin-tidal coupling on the resonance frequency. We accomplish these aims by considering the response function, the frequency-dependent ratio of the induced quadrupole to the tidal field, which encodes these effects. Having calculated the response function, we first match the spin-tidal coupling constant to numerical results for the $f$-mode frequency of isolated spinning neutron stars. We then find a quasiuniversal relation for this coupling, i.e., a relation that is approximately independent of the equation of state for the nuclear matter. Subsequently, we identify relativistic effects due to redshift and frame dragging on the resonance frequency in a binary and quantify their importance.

\section{A. Tidal response function}

To compute the response function, it is easiest to work in the Fourier domain. We take the Fourier transform, denoted by a tilde, of the dynamical tidal variables according to the conventions

$$
Q_{m}(t)=\int \frac{d \omega}{2 \pi} \tilde{Q}_{m}(\omega) e^{-i \omega t},
$$

and similarly for $E_{m}$. It is now straightforward to solve the equation of motion in spherical-harmonic basis (4.14) for $\tilde{Q}_{m}(\omega)$,

$$
\tilde{Q}_{m}=-\tilde{F}_{m} \tilde{E}_{m}
$$

with $\tilde{E}_{m}=2 \pi \mathcal{E}_{m} \delta\left(\omega-m \omega_{\text {orb }}\right)$. We find that the gravitoelectric quadrupolar frequency-domain tidal response is given by

$$
\tilde{F}_{m}=\frac{z^{2} \omega_{0}^{2} \lambda_{0}}{z^{2} \omega_{0}^{2}-\left[\omega-m \Omega_{\mathrm{FD}}+m z \bar{C}_{S Q} S_{1}\right]^{2}} .
$$

For the limiting case of adiabatic tides $\omega \sim m \omega_{\text {orb }} \rightarrow 0$ the response function reduces to the tidal deformability $\tilde{F}_{m}(\omega=$ $0)=\lambda_{0}$, noticing that $\Omega_{\mathrm{FD}}(x=0)=0$ and that we neglect terms quadratic in $S_{1}$. Hence, the response function is a generalization of the Love number $\lambda_{0}$ to dynamical frequencydependent tides. The poles of the response correspond to a resonant excitation of the $f$ mode. We will exploit this fact in our analyses below.

At this point it is convenient to pick a sign convention for the frequencies. For our purpose, it is most useful to assume a fixed sign of the orbital driving frequency as $\omega_{\text {orb }}>0$ and allow for both signs for the spin $S_{1}$ encoding its orientation (aligned $S_{1}>0$ or antialigned $S_{1}<0$ ). Since $\omega=m \omega_{\text {orb }}$ it follows that $\omega \lessgtr 0$ for $m \lessgtr 0$, or $|\omega|=\operatorname{sgn}(m) \omega$. Prograde and retrograde motion of the tidal bulge (relative to the neutron star spin) corresponds to $\operatorname{sgn}(m)= \pm \operatorname{sgn}\left(S_{1}\right)$.

\section{B. Matching the spin-tidal coupling}

To determine the spin-tidal coupling coefficient $\bar{C}_{S Q}$, it is sufficient to consider an isolated neutron star without a companion. For such a star the redshift reduces to $z=1$ and the frame dragging vanishes $\Omega_{\mathrm{FD}}=0$. The poles of the response (5.3) are located at frequencies $\omega$ equal to the inertial-frame $f$-mode frequency $\omega_{f}>0$ of the spinning neutron star, i.e., at $|\omega|=\omega_{f}$. This leads us to the identification of the spin-induced shift of the $f$-mode frequency

$$
\Delta \omega_{0} \equiv \omega_{f}-\omega_{0}=-|m| \bar{C}_{S Q} S_{1},
$$

recalling that $\omega_{0}$ is the $f$-mode frequency (or pole of the response) of a nonspinning star. In terms of the constant $C_{S Q}$ in Eq. (3.14) it holds $\Delta \omega_{0}-|m| \Omega_{1}=-|m| C_{S Q} S_{1}$, which is the corotating-frame frequency shift; the corotating-frame $f$ mode frequency reads as $\tilde{\omega}_{f}=\omega_{f}-|m| \Omega_{1}$ and $S_{1}=I \Omega_{1}$. In the remainder of this section we will drop the label 1 on $\Omega$; the meaning that it is the rotation frequency of the extended body will be implied.

To fix the relativistic value of the spin-tidal coupling $\bar{C}_{S Q}$, we compare the effective frequency shift from Eq. (5.4) to results for the $f$-mode frequencies of rotating relativistic NSs in the Cowling approximation from Ref. [201] (see also [202,203]), specializing to the slow-rotation regime. Specifically, in Ref. [201], Doneva et al. provide quadratic ploynomial fits for the $|m|=\ell f$-mode frequencies in the corotating frame for the stable $(s)$ and unstable $(u)$ branches of the form

$$
\frac{\tilde{\omega}_{\ell}}{\omega_{0}}=1+a_{\ell}^{s / u}\left|\frac{\Omega}{\Omega_{K}}\right|-b_{\ell}^{s / u}\left|\frac{\Omega}{\Omega_{K}}\right|^{2} .
$$

Note that the $\tilde{\omega}_{\ell}$ are defined to be positive, just like our $\omega_{f}$. In our convention, the stable (prograde) branch corresponds to $\Omega>0$ and the unstable (retrograde) one to $\Omega<0$. The coefficients were determined in Eqs. (21)-(24) of Ref. [201] to be $a_{2}^{u}=0.402, b_{2}^{u}=-0.406, a_{3}^{u}=0.373, b_{2}^{u}=-0.485$, and $a^{s}=-0.235, b^{s}=-0.358$ for both $\ell=2,3$. The parameter $\Omega_{K}$, the Kepler frequency, was found to be well approximated by $\Omega_{K}[\mathrm{kHz}]=2 \pi\left[1.716 \sqrt{\bar{\rho}_{0}}-0.189\right]$, where $\bar{\rho}_{0}=\left(m_{1} / 1.4 M_{\odot}\right) /(R / 10 \mathrm{~km})^{3}$ is the scaled mean density of the nonrotating background solution with mass $m_{1}$ and radius $R$. Using the transformation to inertial-frame frequencies $\omega_{\ell}=\tilde{\omega}_{\ell}+|m| \Omega$ (which actually flips the sign of the frequency shift), we find the spin-induced shift of the frequency $\Delta \omega_{0}=\omega_{\ell=2}-\omega_{0}$ and hence a matching for the spin-tidal coupling (recalling $|m|=\ell=2$ ):

$$
\bar{C}_{S Q}=\frac{\omega_{0}-\tilde{\omega}_{\ell=2}-2 \Omega}{2 I \Omega} .
$$

To find a constant value for $\bar{C}_{S Q}$, one should take here the limit of small rotation frequency $\Omega_{1} \equiv \Omega \rightarrow 0$. We note that we obtain $\omega_{0}$ using our own code in this paper, solving the linear perturbation equations of nonrotating neutron stars (without making use of the Cowling approximation). A quasiuniversal fit for $\Delta \omega_{0}$ is also given in Eq. (4) of Ref. [204], which does not make use of the Cowling approximation, but is restricted so far to $\ell=2$.

\section{Universality of the coupling}

We find that the spin-tidal coupling fulfills a quasiuniversal relation that is approximately independent of the (realistic) neutron star equation of state and given by

$$
\bar{C}_{S Q} \approx-\frac{3}{4 I} \text { or } \quad \Delta \omega_{0} \approx \frac{3}{2} \Omega
$$


For the purpose of checking this relation, we calculate $\Omega_{K}$ using the RNS code [205,206] and $\omega_{0}$ from the perturbation equations of nonrotating NSs (specifically the version given in Ref. [190]) for the MS1b and SLy equations of state and neutron star masses ranging between $1.1-2.0 M_{\odot}$. Inserting these values for $\Omega_{K}$ and $\omega_{0}$ into above fit (5.5) leads to $\bar{C}_{S Q}$ via Eq. (5.6). Note that the stable and unstable branches are described by different signs of $\Omega$ here and are averaged over to arrive at Eq. (5.7). This symmetry between stable and unstable branches in the linear regime, which can be inferred from Eq. (5.4) here, is not manifest in the fit (5.5), which is based on data points that are mostly in the regime nonlinear in $\Omega$. Clearly, it would be desirable to check Eq. (5.7), which describes the linear regime, within a slow rotation approximation in the future (and without making use of the Cowling approximation). Note that also the assumption of linear tides could bias the predicted $f$-mode frequency in a similar manner as the Cowling approximation and should be investigated further in the future; see, e.g., Ref. [207] for a recent study.

Note that Eq. (5.7) is consistent with the Newtonian case considered in Ref. [208]. We also checked our relation against the updated fit in Eq. (4) of Ref. [204], which leads to $\Delta \omega_{0} \approx 2 \pi\left|a_{1}^{s / u}\right| \Omega$ and it holds $2 \pi\left|a_{1}^{s / u}\right|=1.2 \ldots 1.4$ which is about $10 \%$ lower than our rough estimate of $\frac{3}{2}$. But still the fit in Ref. [204] might not be optimal in the slow-rotation regime (i.e., most data points are for fast rotation). However, Ref. [204] provides an optimal extension of the quasiuniversal relation above to fast rotation for $\ell=2$.

Finally, we also consider the shift in the octopole $(\ell=3)$ sector, which we estimate through a similar procedure as for the quadrupole explained above. We find that the octopole $f$ mode frequency $\omega_{03}$ is effectively shifted by

$$
\Delta \omega_{03} \approx \frac{5}{2} \Omega .
$$

In order to obtain definite values for the frequency shift given the spin $S=I \Omega$, we also need to know the moment of inertia $I$. For neutron stars, the moment of inertia is related to the dimensionless Love numbers $\Lambda=\lambda_{0} / m_{1}^{5}$ through a nearly universal relation (that holds over a wide range of equations of state) of the form

$$
\ln (I) \approx \sum_{i=0}^{4} c_{i}(\ln \Lambda)^{i},
$$

where the coefficients are given in Table I of Ref. [209] as $c_{0}=1.496, c_{1}=0.05951, c_{2}=0.02238, c_{3}=-6.953 \times$ $10^{-4}$, and $c_{4}=8.345 \times 10^{-6}$.

\section{Relativistic effects on the resonance frequency}

Let us now investigate the $f$-mode resonances in a binary system. As for the spin effects, we will use the poles in $\omega$ of the response function (5.3) to determine these effects. The driving force $\tilde{E}_{m}$ can only excite a resonance for $|m|=2$. Using $\omega=m \omega_{\text {orb }}$, this leads to the resonance condition

$$
\omega_{\mathrm{orb}}-\Omega_{\mathrm{FD}}=z \frac{\omega_{f}}{2} \quad \text { (at resonance). }
$$

We can interpret this in the following way: The framedragging frequency effectively shifts the orbital frequency

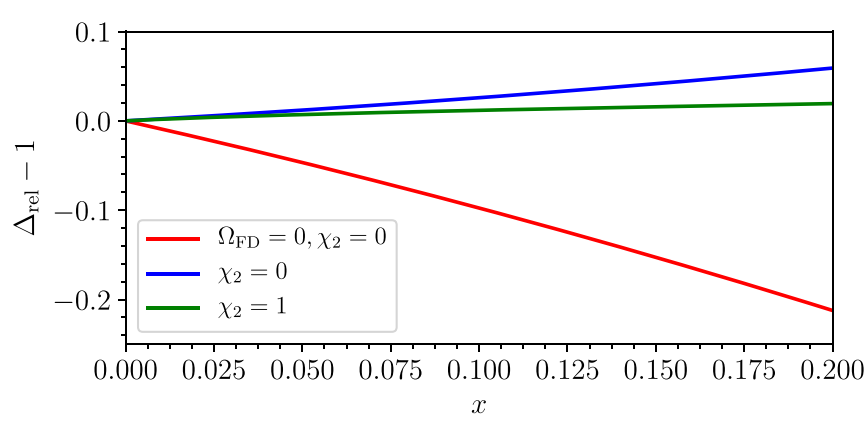

FIG. 1. Relativistic effects on the resonance frequency (5.11) for mass ratio $q=m_{2} / m_{1}=2$. The red curve shows only the redshift effect, the blue curve neglects the companion spin, and the green curve is with "maximal" aligned spin on the companion. The effect of the black-hole spin becomes larger with increasing mass ratio, and is opposite for antialigned spin.

that the neutron star experiences, while the redshift factor effectively reduces the mode frequency.

In the absence of relativistic redshift and frame-dragging effects, the resonance happens at an orbital frequency of $\omega_{f} / 2$. Thus, it makes sense to normalize the relativistic resonant frequency $\omega_{\text {orb }}=\omega_{\text {orb }}^{\text {res }}$ from Eq. (5.10) as

$$
\begin{aligned}
\Delta_{\mathrm{rel}} & \equiv \frac{2 \omega_{\mathrm{orb}}^{\mathrm{res}}}{\omega_{f}}=\frac{z}{1-\Omega_{\mathrm{FD}} / \omega_{\mathrm{orb}}} \\
& =1+v x-\chi_{2} X_{2}^{2} x^{3 / 2}+\frac{9+4 v}{6} v x^{2}+O\left(x^{5 / 2}\right),
\end{aligned}
$$

such that $\Delta_{\text {rel }} \approx 1$ at the resonance in the absence of relativistic effects. The result for the relativistic shifts of the resonance in terms of $\Delta_{\text {rel }}$ are displayed in Fig. 1; see Sec. I.B of Ref. [173] for a detailed interpretation.

We see in Fig. 1 that the redshift (red curve) and framedragging effects almost cancel out (blue curve almost at $\Delta_{\text {rel }} \approx 1$ ) for comparable-mass binaries. This was already noted qualitatively in Ref. [173], and is now quantified by Eq. (5.11). We note that numerical simulations of eccentric binaries, e.g., [210], found that the radiation emanating from the neutron star oscillations shows only the redshift but no noticeable frame-dragging effects. This is not immediately in conflict with our observation, which considers the orbital frequency (and radiation produced by the orbital motion), but it would be desirable to investigate relativistic effects on the radiation emanating from the neutron star oscillations analytically in future work.

The frame dragging generated by the companion spin also shifts the resonance frequency, but this effect is small for comparable-mass binaries (see the discussion above regarding the sign of this dragging). This changes with increasing mass of the companion, such that the companion spin can dominate over the orbital angular momentum. For larger mass blackhole companions, however, the net effect of tidal interactions decreases and becomes more difficult to discern. To conclude, for a large part of the binary parameter space relevant for neutron stars, we can approximately neglect the relativistic effects on the resonance $\Delta_{\text {rel }} \approx 1$. They would be important for broader applications to black-hole mimickers and waveform models for third-generation detectors, which is outside 
the scope of this paper. For neutron star binaries, the dominant effect on the resonance is due to the spin-tidal coupling (5.4). We hence proceed in the next section with a Newtonian approximation and incorporate the spin-tidal interaction in the effective Love number introduced in Refs. [172,173].

\section{ADAPTING THE SEOBNRv4T MODEL}

In the preceding section, we identified the spin-tidal coupling and the corresponding shift of the tidal-resonance frequency as the most important spin effect on dynamical tides. In this section, we incorporate this spin-tidal coupling in the SEOBNRv4T model. We have implemented these modifications in the LIGO Algorithms Library LALSuite. ${ }^{3}$ In this model, the dynamical $f$-mode tidal effects are included through an effective Love number, calculated in the Newtonian limit, that approximately captures the frequency dependence of the response [172,173]. The model is still relativistic since it utilizes post-Newtonian results for the tidal interaction $\sim E_{\mu \nu} E^{\mu \nu}$, currently to next-to-next-to leading order [191-194]. The effective Love number is calculated from an analysis of the solution for the oscillator amplitudes $Q_{m}$ before and during the $f$-mode resonance. Below, we discuss the main modifications to this due to the Coriolis effect. A comparison to related work in Ref. [104] is given in Appendix A.

We first consider the solutions for $Q_{m}$ before the resonance. ${ }^{4}$ We note that the $m=0$ mode has a vanishing frequency at linear order in spin and cannot be resonantly excited. Furthermore, for $|m|=1$ the driving force (4.15) vanishes. Thus, the only contributions to the resonance are associated with $|m|=2$. Gathering the preresonance solution (where $\omega_{\text {orb }} \approx$ const) for $Q_{2}=Q_{-2}^{*}$ from Eqs. (5.2), (4.15), and (5.3), neglecting relativistic effects from redshift $z \approx 1$ and frame dragging $\Omega_{\mathrm{FD}} \approx 0$ (as justified in the previous section), and transforming back to the time domain leads to

$$
Q_{2}=\frac{-\lambda_{0} \omega_{0}^{2} \mathcal{E}_{2} e^{-2 i \omega_{\mathrm{orb}} t}}{\omega_{0}^{2}-\left(2 \omega_{\mathrm{orb}}-\Delta \omega_{0}\right)^{2}},
$$

with $\Delta \omega_{0}=-2 \bar{C}_{S Q} S_{1}$ [see Eq. (5.4)]. Note that although we are assuming that $\Delta \omega / \omega_{0}$ is small, we do not expand the denominator in the solution. This is important to preserve the underlying physics of the resonance shift, and is a common approach for oscillators with small perturbations to their equations of motion (e.g., for an anharmonic oscillator [211]).

\footnotetext{
${ }^{3}$ https://github.com/jsteinhoff/lalsuite/tree/tidal_resonance_ NSspin.

${ }^{4}$ Our previous work [172,173] used a different notation for quadrupole components, given by

$$
\left(Q^{i j}\right)=\left(\begin{array}{ccc}
\alpha+b & c & 0 \\
c & \alpha-b & 0 \\
0 & 0 & -2 \alpha
\end{array}\right) .
$$

These variables are related to the $(2, m)$ degrees of freedom by $\alpha=$ $-Q_{0} / \sqrt{6}, b=\left(Q_{2}+Q_{-2}\right) / 2=\operatorname{Re}\left(Q_{2}\right)$, and $c=i\left(Q_{2}-Q_{-2}\right) / 2=$ $-\operatorname{Im}\left(Q_{2}\right)$.
}

Near the resonance, the denominator of the solutions (6.1) vanishes and the dynamics requires a local analysis that accounts for the evolution of $\omega_{\text {orb }}$ due to gravitational radiation. This was discussed for the nonspinning case in [172,173] in terms of two-timescale expansions that exploit the hierarchy between the timescales in the system associated with the orbital motion $\sim \omega_{\text {orb }}^{-1}$, the $f$-mode oscillations $\sim \omega_{0}^{-1}$, and the gravitational radiation reaction $\tilde{t}=\epsilon \phi$, where $\phi=\int \omega_{\text {orb }} d t$ and $\epsilon=256 \mu M^{2 / 3}\left(\omega_{f} / 2\right)^{5 / 3} / 5$ is a small dimensionless parameter; the temporal width of the resonance is intermediate between the orbital and radiation reaction timescales. Here, we promote these nonspinning results to the spinning case with minor but important modifications. We will obtain approximate results of the dominant effect without redoing the entire calculations by using different physical perspectives of the Coriolis effect for the analysis away from and near the resonance, as we now discuss. The asymptotic behavior of the solution (6.1) near the resonance is

$$
\lim _{\omega_{\mathrm{orb}} \rightarrow \frac{\omega_{0}+\Delta \omega_{0}}{2}} Q_{2}=-\frac{\lambda_{0} \overline{\mathcal{E}} e^{2 i\left(\tilde{t}_{\mathrm{res}} / \epsilon+\hat{t} / \sqrt{\epsilon}\right)}}{2 \sqrt{\epsilon} \hat{t}\left|\bar{\Omega}^{\prime}\right|}+O\left(\hat{t}^{-3}, \epsilon^{0}\right) .
$$

Here, $\hat{t}=\sqrt{\epsilon}\left(\phi-\phi_{\text {res }}\right)$ is a rescaled shifted phase variable and "res" denotes evaluation at the resonance. The quantity $\overline{\mathcal{E}}=-\mathcal{E}_{2} \omega_{0}^{2} /\left(2 \omega_{\text {orb }}-\Delta \omega_{0}\right)^{2}$ is a rescaled tidal amplitude. The function $\bar{\Omega}$ is the frequency ratio between the $f$-mode and tidal driving frequencies. Its rescaled derivative evaluated at the resonance is given by

$$
\begin{aligned}
\bar{\Omega}^{\prime} & =\left.\frac{d}{d \tilde{t}}\left(\frac{\omega_{0}}{2 \omega_{\text {orb }}-\Delta \omega_{0}}\right)\right|_{\text {res }} \\
& =\left.\frac{\dot{\omega}_{\text {orb }}}{\epsilon \omega_{\text {orb }}} \frac{\partial}{\partial \omega_{\text {orb }}}\left[\frac{\omega_{0}}{2 \omega_{\text {orb }}-\Delta \omega_{0}}\right]\right|_{\omega_{\text {orb }}=\left(\omega_{0}+\Delta \omega_{0}\right) / 2}
\end{aligned}
$$

In the nonspinning case when $\Delta \omega_{0}=0$, this expression evaluates to be $\bar{\Omega}^{\prime}=-\frac{3}{8}$ using the leading-order frequency evolution due to gravitational radiation reaction $\dot{\omega}_{\text {orb }}=$ $96 / 5 \mu M^{2 / 3} \omega_{\text {orb }}^{11 / 3}$. In the spinning case, however, there is an extra contribution that depends on the frequency shift and we obtain

$$
\bar{\Omega}^{\prime}=-\frac{3}{8} \frac{\omega_{0}+\Delta \omega_{0}}{\omega_{0}} .
$$

Next, we consider the solutions in the resonance region. In this regime, the Coriolis effect can be viewed as an effective shift in the $f$-mode frequency. This means that all the results from [173] carry over in a straightforward manner with the only change being a shift in $\omega_{f}$. The inner solutions are thus given by

$$
\begin{aligned}
Q_{2}^{\mathrm{res}}= & \frac{\lambda_{0} \overline{\mathcal{E}} e^{2 i \phi}}{\sqrt{\epsilon}}\left[\cos \left(\left|\tilde{\Omega}^{\prime}\right| \hat{t}^{2}\right) \int_{-\infty}^{\hat{t}} \sin \left(\left|\tilde{\Omega}^{\prime}\right| s^{2}\right) d s\right. \\
& \left.-\sin \left(\left|\tilde{\Omega}^{\prime}\right| \hat{t}^{2}\right) \int_{-\infty}^{\hat{t}} \cos \left(\left|\tilde{\Omega}^{\prime}\right| s^{2}\right) d s\right] .
\end{aligned}
$$

The ratio of mode and tidal forcing frequencies in the nearresonance region is

$$
\tilde{\Omega}=\frac{\omega_{0}+\Delta \omega_{0}}{2 \omega_{\text {orb }}}
$$


and thus $\tilde{\Omega}^{\prime}=-\frac{3}{8}$ as in the nonspinning case since a modification of $\omega_{0}$ does not affect the derivatives. The asymptotic behavior of this solution away from the resonance is

$$
\lim _{\omega_{\text {orb } \rightarrow} \rightarrow \frac{\omega_{0}+\Delta \omega_{0}}{2}} Q_{2}^{\mathrm{res}}=-\frac{\lambda_{0} \overline{\mathcal{E}} e^{2 i\left(\tilde{t}_{\mathrm{res}} / \epsilon \hat{t} / \sqrt{\epsilon}\right)}}{2 \sqrt{\epsilon} \hat{t}\left|\tilde{\Omega}^{\prime}\right|}+O\left(\hat{t}^{-3}, \epsilon^{0}\right) .
$$

We see from the expansions (6.2) and (6.7) that the outer (6.1) and inner (6.5) solutions match provided that we also introduce a shift in $\lambda_{0}$ in the near-resonance solutions given by

$$
\lambda_{0}^{\text {near-res }}=\frac{\lambda_{0}}{1+\Delta \omega_{0} / \omega_{0}} .
$$

Note that as above we did not expand the denominator in $\Delta \omega_{0}$ in Eq. (6.8) to guarantee the matching. Finally, we can write the composite solution for the quadrupole by combining the preresonance and near-resonance solutions with the above modifications in each regime and subtracting their common singularity, as explained in Ref. [173]. The last step is to ensure the correct limit at low frequencies $\omega_{\text {orb }} / \Delta \omega_{0} \ll 1$ by including an overall factor of $\left(1-\Delta \omega_{0}^{2} / \omega_{0}^{2}\right)$. We then compute the effective Love number $\Lambda_{\ell}=\lambda_{0 \ell} \hat{k}_{\ell}^{\text {eff }} / m_{1}^{5}$ used in the EOB code from

$$
\hat{k}_{\mathrm{eff}}=-\frac{Q_{i j} E_{i j}}{\lambda_{0} E_{k l} E_{k l}}=-\frac{\sum_{m} Q_{m} E_{m}}{\lambda_{0} \sum_{m} E_{m} E_{m}} .
$$

We display the results here with the convention that the sign of $\Delta \omega_{0 \ell}$ depends on the spin orientation and that it is a function of the EOB coordinate $r$ through $\omega_{\text {orb }}=M^{1 / 2} r^{-3 / 2}$. The dynamical tidal enhancement factor including the shifts is then given by

$$
\begin{aligned}
\hat{k}_{\ell}^{\mathrm{eff}}= & a_{\ell}+b_{\ell}\left(1-\frac{\left(\Delta \omega_{0 \ell}\right)^{2}}{\omega_{0 \ell}^{2}}\right)\left\{\frac{\omega_{0 \ell}^{2}}{\omega_{0 \ell}^{2}-\left(\ell \omega_{\mathrm{orb}}-\Delta \omega_{0 \ell}\right)^{2}}\right. \\
& +\left[\frac{\omega_{f, \ell}^{2}}{2 \sqrt{\epsilon_{\ell}} \hat{t}\left|\tilde{\Omega}^{\prime}\right|\left(1+\frac{\Delta \omega_{0 \ell}}{\omega_{0 \ell}}\right)\left(\ell \omega_{\mathrm{orb}}\right)^{2}}\right]_{\omega_{f, \ell}=\omega_{0 \ell}+\Delta \omega_{0 \ell}} \\
& \left.+\left[\frac{\omega_{f, \ell}^{2}}{\sqrt{\epsilon_{\ell}}\left(\ell \omega_{\mathrm{orb}}\right)^{2}\left(1+\frac{\Delta \omega_{0 \ell}}{\omega_{0 \ell}}\right)} \mathcal{Q}_{\ell \ell}(\hat{t})\right]_{\omega_{f, \ell}=\omega_{0 \ell}+\Delta \omega_{0 \ell}}\right\},
\end{aligned}
$$

where

$$
\begin{aligned}
\mathcal{Q}_{\ell \ell}(\hat{t})= & \cos \left(\left|\tilde{\Omega}^{\prime}\right| \hat{t}^{2}\right) \int_{-\infty}^{\hat{t}} \sin \left(\left|\tilde{\Omega}^{\prime}\right| s^{2}\right) d s \\
& -\sin \left(\left|\tilde{\Omega}^{\prime}\right| \hat{t}^{2}\right) \int_{-\infty}^{\hat{t}} \cos \left(\left|\tilde{\Omega}^{\prime}\right| s^{2}\right) d s .
\end{aligned}
$$

The quantities $\hat{t}\left(\omega_{f}\right)$ and the dimensionless parameter $\epsilon_{\ell}\left(\omega_{f}\right)$ are given as explicit functions of $r$ by

$\hat{t}=\frac{8}{5 \sqrt{\epsilon_{\ell}}}\left(1-\frac{r^{5 / 2} \omega_{f, \ell}^{5 / 3}}{\ell^{5 / 3} M^{5 / 6}}\right), \quad \epsilon_{\ell}=\frac{256 \mu M^{2 / 3} \omega_{f, \ell}^{5 / 3}}{5 \ell^{5 / 3}}$.

In Eq. (6.10) a body label on the quantities $\omega_{0 \ell}, \hat{t}, \epsilon_{\ell}$, and $\mathcal{Q}_{\ell \ell}$ is implied. For each $\ell$ multipole only $|m|=\ell$ contributes in Eq. (6.10) because the effect of modes with $|m| \neq \ell$ has

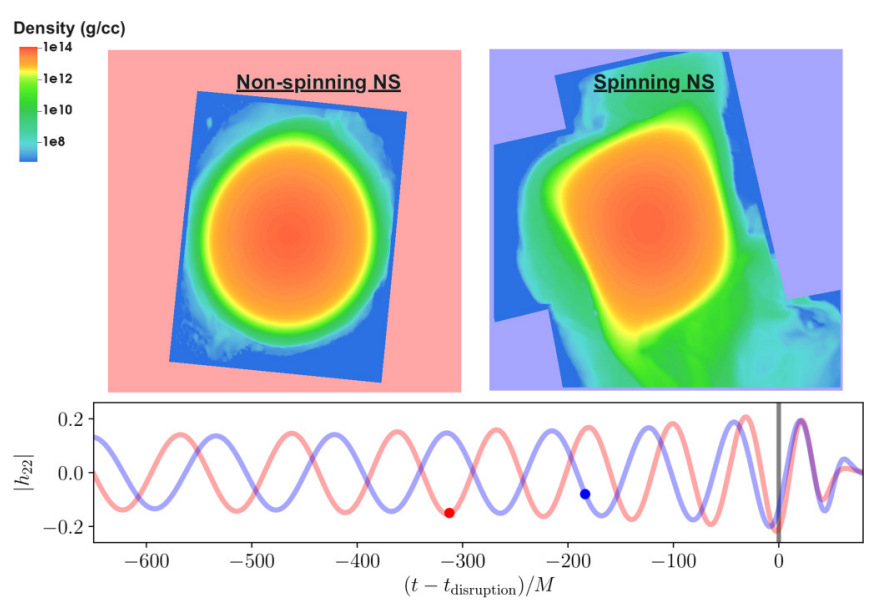

FIG. 2. Matter density in the late inspiral for the nonspinning $q=1 \mathrm{NSBH}$ simulation (top left), and for the simulation with a spinning neutron star (top right), as well as the corresponding GW signal. We show the density in the equatorial plane at a time when the binary separation is $\sim 35 \mathrm{~km}$ (cf. markers in the bottom panel). The differences in the amount of distortion in the matter distributions (larger for the spinning NS) and onset of tidal disruption (earlier for the spinning NS), while being purely gauge dependent, can be considered as a visualization of the enhancement of nonequilibrium tides. The waveforms are aligned at the tidal disruption as determined from the peak GW amplitude.

already been taken into account as adiabatic contributions. For the quadrupole and octupole multipole moments the coefficients are given by $\left(a_{2}, a_{3}\right)=\left(\frac{1}{4}, \frac{3}{8}\right)$ and $\left(b_{2}, b_{3}\right)=\left(\frac{3}{4}, \frac{5}{8}\right)$.

\section{COMPARISONS TO NUMERICAL-RELATIVITY SIMULATIONS}

In this section, we compare the performance of our extension of the SEOBNRv4T approximant derived in Sec. VI with numerical-relativity waveforms. We will focus on black-hole-neutron star (NSBH) systems simulated with the SPECcode [212-214] and on binary neutron star (NSNS) simulations with the BAM code [215-217].

In addition to the quantitative comparison that we will show in the next subsections, numerical-relativity simulations provide also a qualitative indication for the importance of nonequilibrium tides. Figure 2 shows a NSBH system with mass ratio $q=m_{2} / m_{1}=1$ and an antialigned neutron star spin, which enhances the excitation of nonequilibrium tides as discussed in Sec. V and can be seen from Eq. (6.10) with a negative $\Delta \omega_{0 \ell}$. While it is difficult to directly quantify these dynamical tides in a gauge-independent manner, the visual difference in Fig. 2 between the spinning and nonspinning matter distributions of the neutron star, which have no invariant meaning, provide an illustration of measurable differences in waveforms that we will analyze below.

\section{A. Comparison to NSBH SXS waveforms}

For an initial comparison to numerical-relativity simulations and a validation of our model, we consider two NSBH setups presented in Ref. [174] and simulated with the SPEC code [212-214]. The two configurations represent an equal- 

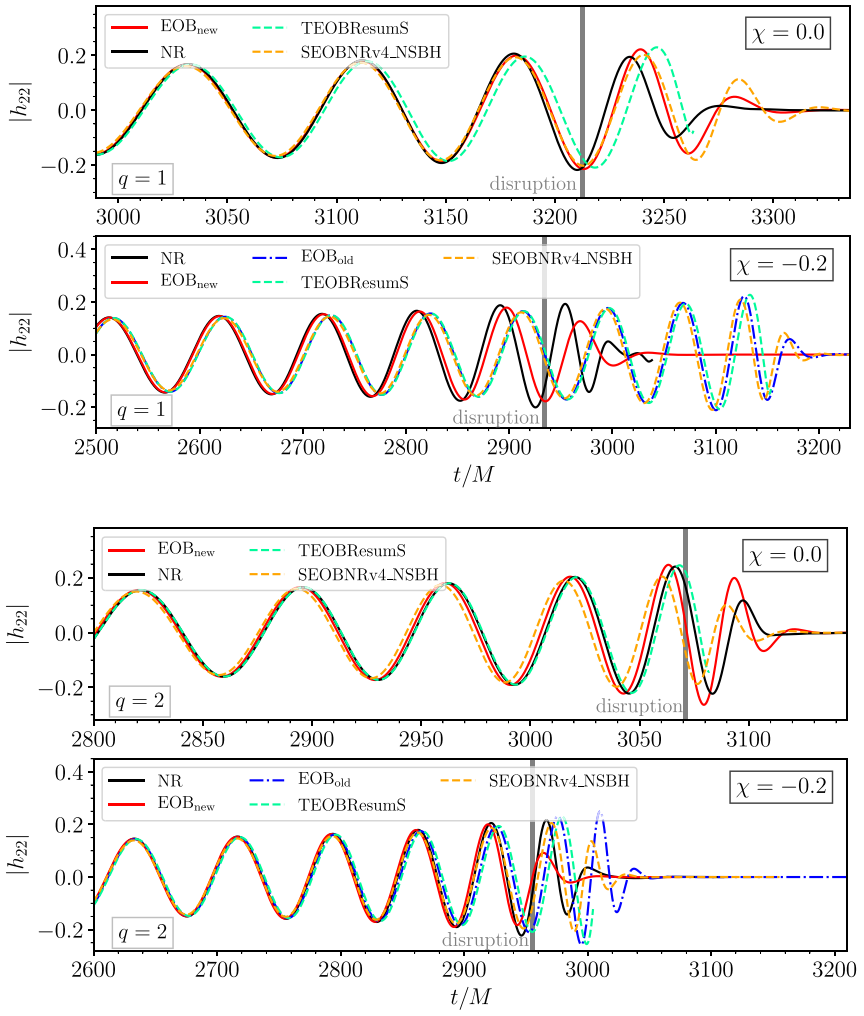

FIG. 3. Comparisons of various models to the NR waveforms for NSBH considered in this work (top, equal mass; bottom, mass ratio $q=2$ ). The TEOBResumS waveforms are tapered to zero after the NSNS merger frequency, and the SEOBNR waveforms are tapered either at the NSNS merger or the $f$-mode resonance, whichever occurs first. Remarkably, the $f$-mode resonance in our model including the spin shifts matches quite well with the tidal disruption frequency in the NR data, while the models that do not include the spin shifts predict a merger at a later time.

mass $q=1$ and an unequal-mass $q=2$ setup employing a single polytropic equation of state $P=\kappa \rho^{2}$, with $\kappa$ chosen so that $m_{1} / R_{1}=0.144$ (e.g.. $R_{1}=13.8 \mathrm{~km}$ if $\left.m_{1}=1.35 M_{\odot}\right)$. Each mass ratio is simulated twice: with a nonspinning neutron star and with an antialigned dimensionless spin $\chi_{1}=$ -0.2 on the neutron star. In both cases the black hole remains nonspinning. The neutron star spins and mass ratios were chosen as examples of a large expected impact of nonequilibrium tides. The numerical-relativity data are publicly available in the SXS catalog [218], where we use the simulations SXS:BHNS:0004 and SXS:BHNS:0005 for the $q=1$ and SXS:BHNS:0002 and SXS:BHNS:0007 for the $q=2$, nonspinning and spinning cases, respectively.

The evolutions start $10-13$ orbits before merger and use eccentricity-reduced $(e<0.002)$, constraint satisfying initial conditions [219,220]. Each case is simulated at three resolutions, and a detailed discussion of the estimated numerical error in these simulations can be found in Ref. [174]. The same error estimates are used in this work. We typically find phase errors of less than $0.1 \mathrm{rad}$ for most of the inspiral and rising to $(0.1-0.2) \mathrm{rad}$ at merger for the $q=1$ cases and to (0.5-1.0) rad for $q=2$.

We show in Fig. 3 the real part of the GW for the dominant $(2,2)$ mode for the two different mass ratios and different spin. The parameters that we use in the EOB models were not obtained from quasiuniversal relations [except for the fit in Eq. (5.5)] and read as

$$
\begin{gathered}
\frac{\lambda_{0}}{m_{1}^{5}}=799.3, \quad m_{1} \omega_{0}=0.06746, \\
\frac{\Delta \omega_{0}}{\omega_{0}}=-0.325, \quad \frac{\lambda_{03}}{m_{1}^{7}}=2246, \\
m_{1} \omega_{03}=0.08805, \quad \frac{\Delta \omega_{03}}{\omega_{03}}=-0.4, \\
C_{E S^{2}}=7.14, \quad \frac{I}{m_{1}^{3}}=15.5,
\end{gathered}
$$

recalling that these are the (quadrupolar, $\ell=2$ ) Love number $\lambda_{0}$, nonspinning $f$-mode frequency $\omega_{0}$, the mode shift $\Delta \omega_{0}$ due to spin, the corresponding octupolar $(\ell=3)$ values $\lambda_{03}, \omega_{03}, \Delta \omega_{03}$, the dimensionless spin-induced quadrupolemoment constant $C_{E S^{2}}$ (normalized to 1 for black holes), and the moment of inertia $I$. We see from these plots that for the nonspinning cases, existing waveform models predict the length of the waveforms and decrease of the GW amplitude due to the tidal disruption of the neutron star to a good approximation. However, for systems with antialigned spins, all existing models, including those specialized for NSBH systems, predict longer waveforms, while our modification with spin effects (red curve) continues to yield a good prediction for the length of the signal. This is because in our model, as also in the nonspinning SEOBNRv4T model, the GW signal is tapered to zero once the system reaches the $f$-mode resonance, if it occurs before the NSNS merger frequency. The fact that for our SEOBNRv4T model the tapering occurs at the $f$-mode resonance for these cases can be seen by comparing to the TEOBResumS results, which are always tapered at the NSNS merger frequency. These findings highlight an interesting point, namely, that there seems to be a direct relation between the $f$-mode resonance and the tidal disruption frequency.

Focusing on the $q=1$ setup, we next show the phase difference between the old (without the Coriolis effect) and our SEOBNRv4T model, as well as results for TEOBResumS [147] and SEOBNSBH [221] in Fig. 4. For the nonspinning case (top panel) all models describe the GW phase accurately up to about one orbit before merger and stay within the estimated uncertainty of the numerical-relativity simulation (shown as the gray shaded region) [174]. Considering the middle panel of Fig. 4, the antialigned spin of the neutron star enhances the dynamical tidal effects. We find that for this setup, the discrepancy in phasing between our SEOBNRv4T model and the numerical-relativity results is significantly less than for the other approximants. Therefore, we find a significantly better performance if nonequilibrium tidal effects are included. Although our version of SEOBNRv4T is outside the estimated numerical uncertainty band close to the tidal disruption, other approximants show a noticeable dephasing even a few orbits before the disruption of the star, and it is this earlier-time regime where we expect to have more analytical control over the physics of the model.

An even more important diagnostic of the robustness of our model, beyond a reduced phase difference in a few example 

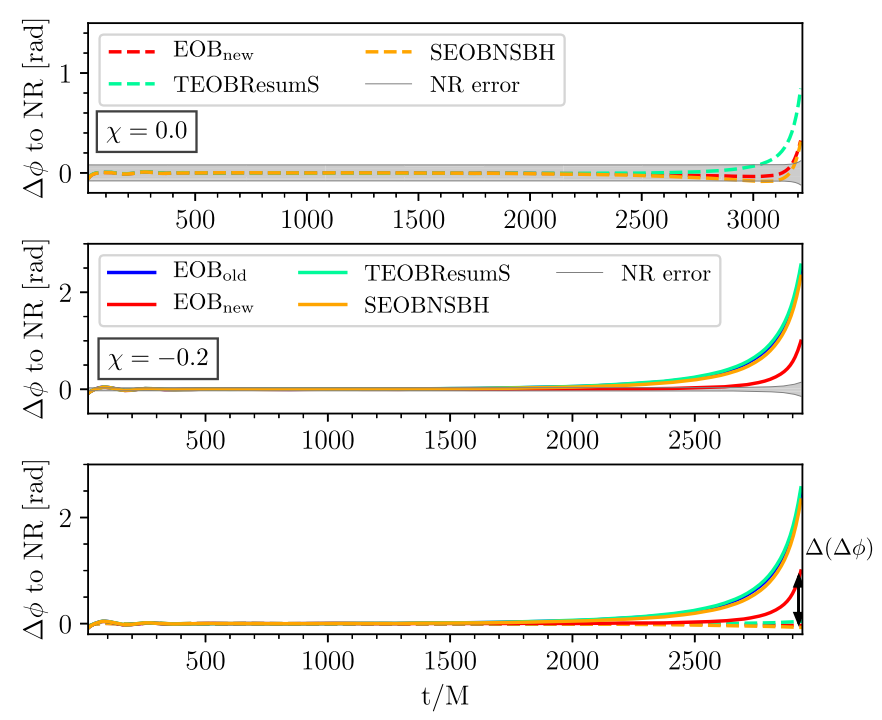

FIG. 4. Phase differences in the NSBH case for different approximants and the numerical-relativity simulations. We consider the difference of the phase difference in the spinning minus the nonspinning case for the $q=1 \mathrm{NSBH}$ simulation described in the main text to quantify systematic dependencies on the spin. The top panel shows phase difference between the numerical-relativity simulation (and its uncertainty shown as a gray band) with the TEOBResumS, SEOBNRv4T, and SEOBNSBH models. The middle panel shows a similar comparison for an antialigned neutron star spin, where we also add our SEOBNRv4T model described in this paper. In the bottom panel, we highlight again the phase difference between the approximants and the simulation results in the spinning and nonspinning cases. The difference between the phase difference, i.e., $\Delta(\Delta \phi)$, is for the our implemented model smallest.

cases, is that the phase differences to the numerical-relativity simulations are consistent between the spinning and nonspinning setups. To test the performance of our model with regards to this criterion we introduce the quantity $\Delta(\Delta) \phi$, which measures the phase difference for the case with spin minus the phase difference in the corresponding nonspinning case. A small $\Delta(\Delta) \phi$ indicates that the physics of the dynamical tidal effects and impact of the Coriolis effect are well captured by the model, up to other physical effects with a different origin that are common among all cases. The definition of $\Delta(\Delta) \phi$ is illustrated in the bottom panel of Fig. 4 showing the phase difference between the EOB model and the SPEC data in the spinning case minus the phase difference for the nonspinning setup, i.e., the smaller the difference the better the consistency between the nonspinning and spinning data.

For a more quantitative presentation, we present $\Delta(\Delta \phi)$ for our and the old SEOBNRv4T models in the top panel of Fig. 5 for the $q=1$ and 2 setups. This clearly shows that for both configurations our model outperforms the previous implementation. Similarly, the bottom panel shows also our model in comparison to other NSBH approximants, where the disagreement between phase difference for spinning and nonspinning with respect to the corresponding numericalrelativity simulations is larger than for the model developed in this paper.
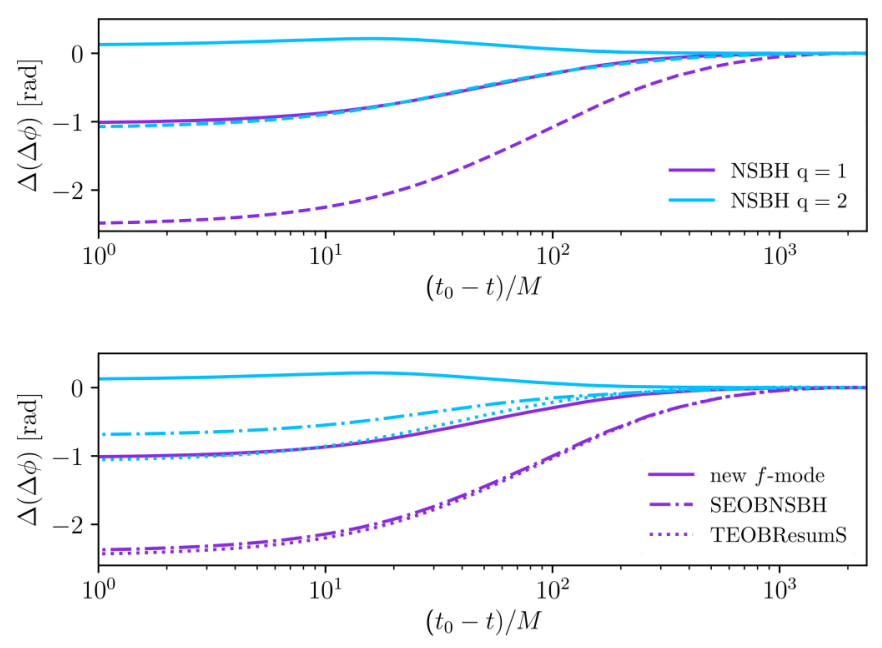

FIG. 5. Phase differences compared to the phase differences in the nonspinning case for the NSBH configurations. Top panel: solid curves are the model developed in this paper, dashed curves are the existing SEOBNRv4T model, which does not account for spin effects on the mode resonances and exhibits a wider spread in phase differences between spinning and nonspinning configurations. Bottom panel: comparison with other waveform models which do not incorporate the spin-induced shift of the $f$ mode. We see that our model has a consistently smaller spread. In each case, $t_{0}$ is the time at which the first merger occurs (i.e., for aligned spins it is the nonspinning merger, and otherwise the merger of the antialigned configuration).

\section{B. NSNS BAM waveforms}

We continue our tests of our model by comparing against numerical-relativity waveforms of NSNS systems computed with the BAM code $[98,128,223,224]$, which include cases with both aligned and antialigned spins. In total, we consider three different equations of state: SLy, H4, MS1b. For all these equations of state, we consider one nonspinning configuration and one to three spinning setups; cf. Table I for further details and for the parameters used in the EOB models. The waveforms from Ref. [223] show a clean second-order convergence, which allows using Richardson extrapolation to obtain a better guess for the true waveform, as discussed in Ref. [225]. We use the Richardson-extrapolated data from Ref. [223] for our comparisons.

For all the cases, we follow a similar procedure as for the NSBH setups by focusing on the difference of the phase difference between the spinning EOB and the numerical-relativity waveforms with respect to their nonspinning counterparts. This way, we explicitly test the imprint of spin on the dynamical tides. Our results are summarized in Fig. 6, where in the top panel the dashed lines refer to the old SEOBNRv4T model without the spin effects on the dynamical tides, and the solid lines show results for our model. We find that for these cases, our model shows a smaller phase difference between the numerical-relativity and the EOB data as the old model. We emphasize that this does not necessarily mean that the total phase difference with respect to the numericalrelativity data decreased in all cases, but rather that the phase difference for the nonspinning and spinning configurations 
TABLE I. NSNS-BAM configurations. The first column defines the name of the configuration with the notation $\operatorname{EOS}_{m^{A}}^{\chi^{A}}$. The subsequent columns describe the EOS [222], the NS' individual masses $m_{A, B}$, the stars' dimensionless spins $\chi_{A, B}$, the Love number $\lambda_{0}$, the nonspinning $f$-mode frequency $\omega_{0}$, the mode shift $\Delta \omega_{0}$ due to spin, the corresponding octupolar $(\ell=3)$ values $\lambda_{03}, \omega_{03}, \Delta \omega_{03}$, the dimensionless spininduced quadrupole-moment constant $C_{E S^{2}}$ (normalized to 1 for black holes), and the moment of inertia $I$. The values here were not obtained from quasiuniversal relations, except for the fit in Eq. (5.5).

\begin{tabular}{|c|c|c|c|c|c|c|c|c|c|c|c|}
\hline Name & EOS & $m_{1,2} / M_{\odot}$ & $\chi_{1,2}$ & $\lambda_{0} / m_{1}^{5}$ & $m_{1} \omega_{0}$ & $\Delta \omega_{0} / \omega_{0}$ & $\lambda_{03} / m_{1}^{7}$ & $m_{1} \omega_{03}$ & $\Delta \omega_{03} / \omega_{03}$ & $C_{E S^{2}}$ & $I / m_{1}^{3}$ \\
\hline $\mathrm{MS} 1 \mathrm{~b}_{1.35}^{-0.10}$ & MS1b & 1.3504 & -0.099 & 1528 & 0.05836 & -0.137 & 4488 & 0.07973 & -0.162 & 8.74 & 18.05 \\
\hline MS1 $1 b_{1.35}^{0.00}$ & MS1b & 1.3500 & +0.000 & 1528 & 0.05836 & +0.000 & 4488 & 0.07973 & +0.000 & & \\
\hline $\mathrm{MS} 1 \mathrm{~b}_{1.35}^{0.10}$ & MS1b & 1.3504 & +0.099 & 1528 & 0.05836 & +0.145 & 4488 & 0.07973 & +0.164 & 8.74 & 18.05 \\
\hline MS1 $b_{1.35}^{0.15}$ & MS1b & 1.3509 & +0.149 & 1525 & 0.05837 & +0.210 & 4474 & 0.07977 & +0.238 & 8.54 & 18.17 \\
\hline $\mathrm{H} 4_{1.37}^{0.00}$ & $\mathrm{H} 4$ & 1.3717 & +0.000 & 1003 & 0.06435 & +0.000 & 2605 & 0.08702 & +0.000 & & \\
\hline $\mathrm{H} 4_{1.37}^{0.14}$ & $\mathrm{H} 4$ & 1.3726 & +0.141 & 1003 & 0.06435 & +0.202 & 2605 & 0.08702 & +0.232 & 7.32 & 16.11 \\
\hline SLy ${ }_{1.35}^{0.00}$ & SLy & 1.3500 & +0.000 & 389.6 & 0.07934 & +0.000 & 705.2 & 0.1067 & +0.000 & & \\
\hline SLy $_{1.35}^{0.05}$ & SLy & 1.3502 & +0.052 & 389.6 & 0.07934 & +0.084 & 705.2 & 0.1067 & +0.096 & 6.18 & 12.34 \\
\hline $\mathrm{SLy}_{1.35}^{0.11}$ & SLy & 1.3506 & +0.106 & 388.8 & 0.07936 & +0.164 & 703.4 & 0.1068 & +0.189 & 5.59 & 12.41 \\
\hline
\end{tabular}

becomes almost identical, indicating that the dependence on parameters is captured well.

The bottom panel of Fig. 6 compares the consistency of various GW models [98,147] between the spinning and the nonspinning configurations for the NSNS binaries. We find that for all the setups our SEOBNRv4T implementation has the smallest $\Delta(\Delta \phi)$, which means that the phase difference between the EOB model and the NR simulation is similar for the spinning and nonspinning cases. These results indicate that (i) the inclusion of spin effects is consistent and (ii) fur-
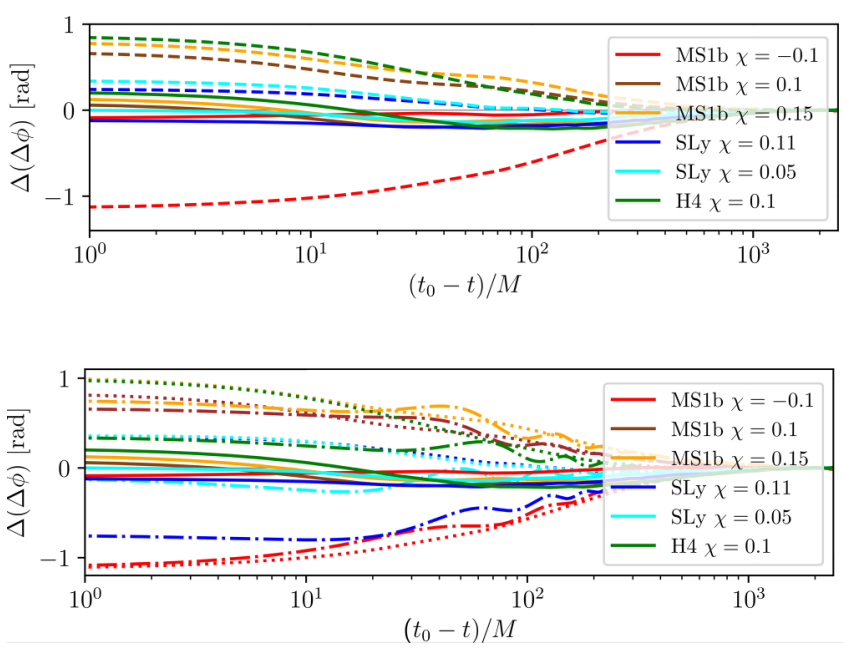

FIG. 6. Phase differences compared to the phase differences in the nonspinning case for the NSNS configurations. Top panel: solid curves are the model developed in this paper, dashed curves are the existing SEOBNRv4T model, which does not account for spin effects on the mode resonances and exhibits a wider spread in phase differences between spinning and nonspinning configurations. Bottom panel: comparison with other waveform models which do not incorporate the spin-induced shift of the $f$ mode. We see that our model has a consistently smaller spread. In each case, $t_{0}$ is the time at which the first merger occurs (i.e., for aligned spins it is the nonspinning merger, and otherwise the merger of the antialigned configuration). ther improvements of the nonspinning sector will likely also improve the agreement between EOB and numerical-relativity predictions for spinning configurations.

\section{CONCLUSIONS}

In this paper, we developed a ready-to-use waveform model that approximately captures the effects of spin on the $f$-mode dynamical tidal response of a neutron star. This model is based on the leading-order terms in a relativistic effective action describing a spinning neutron star in a binary system, which we derived.

We found that within our approximation, a nonvanishing spin gives rise to a Coriolis interaction term in the action. We determined the coupling coefficient for this term from the spin-induced shift of the $f$-mode frequencies in slowly rotating relativistic neutron stars. A quasiuniversal relation for this coupling coefficient was found as well, which is important for reducing the number of parameters to be inferred from GW observations. Further, using explicit post-Newtonian results we also analyzed relativistic effects (redshift and frame dragging) on the dynamical tidal response and found that they are subdominant compared to the spin effects.

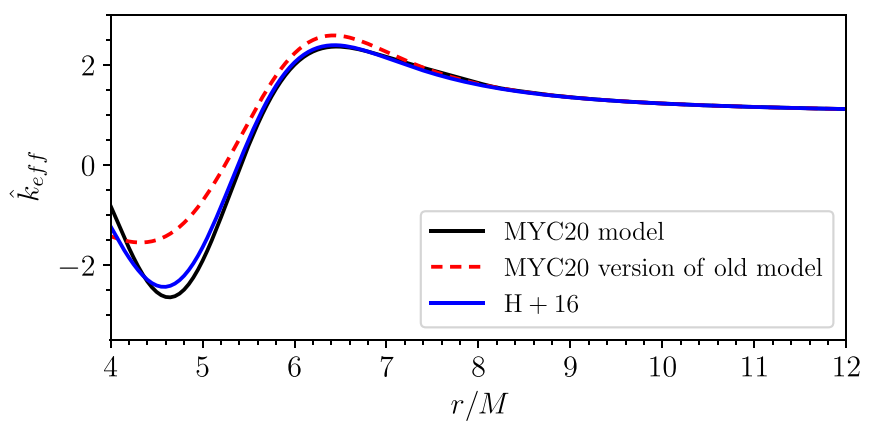

FIG. 7. Analytical approximations for a nonspinning equal-mass binary ( $\left.\mathrm{H} 4 \mathrm{EoS}, m=1.4 M_{\odot}\right)$ with one extended body. Here, MYC20 is from Eq. (54) with the higher-order matching from Eq. (55) in Ref. [104]. 
We then developed a simple model that captures the main Coriolis effects on dynamical tides and incorporated it into a state-of-the-art EOB model. To test this model, we performed comparisons to results from numerical-relativity simulations of binary neutron star and neutron star-black-hole binaries. Our model showed improved behavior over the parameter space compared to existing models that neglect the Coriolis effect. Moreover, we found that it predicted the tidal disruption frequency in mixed binaries significantly better than models without this spin-tidal effect. Our model is implemented in the LIGO Algorithms library.

This work also identified important directions for future work. Moreover, the results from this paper provide a useful foundation for including these spin-tidal effects also in other waveform models. Improving the physics content of models is important for accurate measurements and robustness over a wider range in parameter space. We have also derived the relativistic effects on the response which can be included in future models, when we will also work out the effective Love number based on the spin-dependent response, allow for misaligned spins and other effects of the companion's spin, and also include other relativistic effects in a binary system. Last but not least, the imprint of the microphysics, in particular of superfluidity [226], on dynamical tides is of high interest for gravitational-wave astronomy.

\section{ACKNOWLEDGMENTS}

We thank G. Creci for checking results in Sec. II. T.H. acknowledges support from the Nederlandse Organisatie voor Wetenschappelijk Onderzoek (NWO) sector plan. F.F. acknowledges support from NASA through Grant No. 80NSSC18K0565, from the DOE through Early Career Award No. DE-SC0020435, and from the NSF through Grant No. PHY-1806278. The authors are grateful for computational resources provided by the LIGO Laboratory and supported by the National Science Foundation Grants No. PHY-0757058 and No. PHY-0823459.

\section{APPENDIX: CONNECTION TO MA, YU, AND CHEN [104]}

Here, we briefly outline similarities and differences with the work of $\mathrm{Ma}$, Yu, and Chen (hereafter MYC20) in Ref. [104], which considered the effect of spin on the $f$ mode in a Newtonian context. The effect of spins on the tidal response in Ref. [104] is interconnected with the orbital dynamics obtained from numerical integrations of the equations of motion for the coupled system of dynamical quadrupoles, orbital variables, and gravitational radiation reaction. For ease of comparing to our results here, we apply the same procedure as for obtaining the effective Love number $\hat{k}_{2}^{\text {eff }}$ oulined in

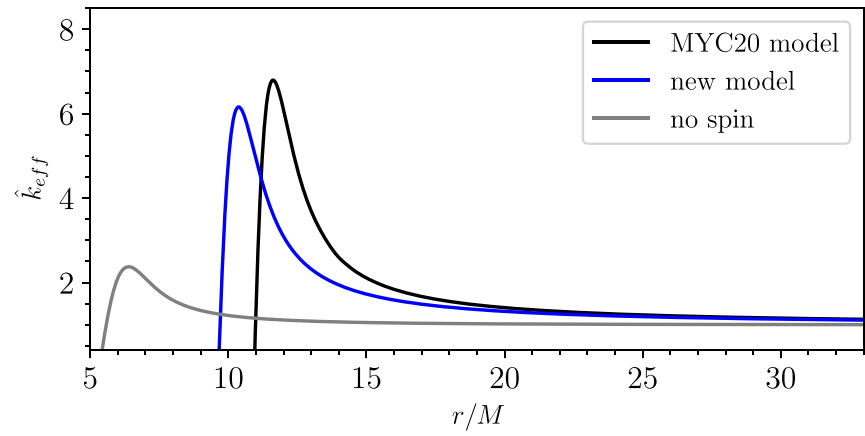

FIG. 8. Comparison between the model developed in this paper with $\left\{\Delta \omega_{0}, \Delta \lambda\right\}$ and MYC20 for a spin frequency $\Omega=2 \pi \times 550 \mathrm{~Hz}$ (H4 EoS, $m=1.4 M_{\odot}, \chi=0.37$ ). For reference, the gray curve indicates the nonspinning result.

Sec. VI to write the formulas in MYC20 explicitly as functions of the orbital separation $r$. The choice of this variable is motivated by the fact that the canonical coordinate $r$ plays a key role for the EOB dynamics, which are the basis of EOB waveforms. The formulas in Ref. [104] are similar to our results, with slight differences in the definitions of variables such as $\hat{t}$ leading to small differences in the response near the resonance, as illustrated in Fig. 7. For instance, in our work, $\hat{t}$ is based on the phase and the parameter $\epsilon$, while in MYC20 $\hat{t}^{Y}$ is based on coordinate time and $\dot{\omega}$ at the resonance time and given explicitly by

$$
\hat{t}^{Y}=\frac{\sqrt{15}}{162^{2 / 3} M^{1 / 3} \sqrt{\mu} w}-\frac{\sqrt{15} r^{4} w}{1282^{1 / 3} M^{5 / 3} \sqrt{\mu}},
$$

where

$$
w=\sqrt{-\omega_{-} \omega_{+}+\Omega^{2}}-\Omega .
$$

Here, $\left|\omega_{ \pm}\right|$are the frequencies of the two branches of $m$ modes whose frequencies coincide for $\Omega=0$.

MYC20 compute the $f$-mode frequencies for Newtonian Maclaurin spheroids. In the nonspinning case, this yields frequencies that are $\sim 320 \mathrm{~Hz}$ smaller than the relativistic values. MYC20 account for this by rescaling the density so as to match the relativistic frequencies. In this paper, we have used the fully relativistic results for the frequencies and their shifts, albeit only within the linear approximation for small $\Omega$. The variables of MYC20 are approximately related to these shifts by $\left|\omega_{ \pm}\right| \approx \omega_{0} \mp \Delta \omega$. These different approximations and prescriptions also affect the orbital frequency at resonance. For instance, for the case of $\Omega=2 \pi 550 \mathrm{~Hz}$ considered in MYC20, which is already outside of the linear regime, the resonance occurs at $\sim 300 \mathrm{~Hz}$ for MTY20 but not until $\sim 350 \mathrm{~Hz}$ for the parameters used here. The resulting predictions for the effective Love number are illustrated in Fig. 8.
[1] J. M. Lattimer and M. Prakash, The equation of state of hot, dense matter and neutron stars, Phys. Rep. 621, 127 (2016).
[2] Edited by L. Rezzolla, P. Pizzochero, D. I. Jones, N. Rea, and I. Vidaña, The Physics and Astrophysics of Neutron Stars, 
Astrophysics and Space Science Library, Vol. 457 (Springer, Cham, 2018), 1st ed.

[3] B. P. Abbott et al. (LIGO Scientific, Virgo), Search for postmerger gravitational waves from the remnant of the binary neutron star merger GW170817, Astrophys. J. Lett. 851, L16 (2017).

[4] B. P. Abbott et al. (LIGO Scientific, Virgo), Properties of the Binary Neutron Star Merger GW170817, Phys. Rev. X 9, 011001 (2019).

[5] B. P. Abbott et al. (LIGO Scientific, Virgo), GW170817: Observation of Gravitational Waves from a Binary Neutron Star Inspiral, Phys. Rev. Lett. 119, 161101 (2017).

[6] A. Bauswein, O. Just, H.-T. Janka, and N. Stergioulas, Neutron-star radius constraints from GW170817 and future detections, Astrophys. J. Lett. 850, L34 (2017).

[7] E. Annala, T. Gorda, A. Kurkela, and A. Vuorinen, Gravitational-Wave Constraints on the Neutron-Star-Matter Equation of State, Phys. Rev. Lett. 120, 172703 (2018).

[8] E. R. Most, L. R. Weih, L. Rezzolla, and J. Schaffner-Bielich, New Constraints on Radii and Tidal Deformabilities of Neutron Stars from GW170817, Phys. Rev. Lett. 120, 261103 (2018).

[9] M. Ruiz, S. L. Shapiro, and A. Tsokaros, GW170817, General Relativistic Magnetohydrodynamic simulations, and the neutron star maximum mass, Phys. Rev. D 97, 021501 (2018).

[10] B. Margalit and B. D. Metzger, Constraining the Maximum Mass of Neutron Stars From Multi-Messenger Observations of GW170817, Astrophys. J. Lett. 850, L19 (2017).

[11] L. Rezzolla, E. R. Most, and L. R. Weih, Using gravitationalwave observations and quasi-universal relations to constrain the maximum mass of neutron stars, Astrophys. J. Lett. 852, L25 (2018).

[12] M. Shibata, S. Fujibayashi, K. Hotokezaka, K. Kiuchi, K. Kyutoku, Y. Sekiguchi, and M. Tanaka, Modeling GW170817 based on numerical relativity and its implications, Phys. Rev. D 96, 123012 (2017).

[13] B. P. Abbott et al. (LIGO Scientific, Virgo), GW170817: Measurements of Neutron Star Radii and Equation of State, Phys. Rev. Lett. 121, 161101 (2018).

[14] S. De, D. Finstad, J. M. Lattimer, D. A. Brown, E. Berger, and C. M. Biwer, Tidal Deformabilities and Radii of Neutron Stars from the Observation of GW170817, Phys. Rev. Lett. 121, 091102 (2018); Tidal Deformabilities and Radii of Neutron Stars from the Observation of GW170817, 121, 259902 (2018).

[15] D. Radice, A. Perego, F. Zappa, and S. Bernuzzi, GW170817: Joint constraint on the neutron star equation of state from multimessenger observations, Astrophys. J. Lett. 852, L29 (2018).

[16] M. W. Coughlin, T. Dietrich, Z. Doctor, D. Kasen, S. Coughlin, A. Jerkstrand, G. Leloudas, O. McBrien, B. D. Metzger, R. O'Shaughnessy et al., Constraints on the neutron star equation of state from AT2017gfo using radiative transfer simulations, Mon. Not. R. Astron. Soc. 480, 3871 (2018).

[17] M. W. Coughlin, T. Dietrich, B. Margalit, and B. D. Metzger, Multimessenger Bayesian parameter inference of a binary neutron star merger, Mon. Not. R. Astron. Soc. 489, L91 (2019).
[18] L. Dai, T. Venumadhav, and B. Zackay, Parameter estimation for GW170817 using relative binning, arXiv:1806.08793.

[19] D. Radice and L. Dai, Multimessenger parameter estimation of GW170817, Eur. Phys. J. A55, 50 (2019).

[20] M. Lucca and L. Sagunski, The lifetime of binary neutron star merger remnants, J. High Energy Astrophys. 27, 33 (2020).

[21] C. D. Capano, I. Tews, S. M. Brown, B. Margalit, S. De, S. Kumar, D. A. Brown, B. Krishnan, and S. Reddy, Stringent constraints on neutron-star radii from multimessenger observations and nuclear theory, Nat. Astron. 4, 625 (2020).

[22] T. Dietrich, M. W. Coughlin, P. T. H. Pang, M. Bulla, J. Heinzel, L. Issa, I. Tews, and S. Antier, Multimessenger constraints on the neutron-star equation of state and the Hubble constant, Science 370, 1450 (2020)

[23] G. Raaijmakers et al., Constraining the dense matter equation of state with joint analysis of NICER and LIGO/Virgo measurements, Astrophys. J. Lett. 893, L21 (2020).

[24] C. Cutler and E. E. Flanagan, Gravitational waves from merging compact binaries: How accurately can one extract the binary's parameters from the inspiral wave form? Phys. Rev. D 49, 2658 (1994).

[25] J. Veitch et al., Parameter estimation for compact binaries with ground-based gravitational-wave observations using the LALInference software library, Phys. Rev. D 91, 042003 (2015).

[26] B. P. Abbott et al. (LIGO Scientific, Virgo), A guide to LIGOVirgo detector noise and extraction of transient gravitationalwave signals, Classical Quantum Gravity 37, 055002 (2020).

[27] B. Carter, Axisymmetric Black Hole Has Only Two Degrees of Freedom, Phys. Rev. Lett. 26, 331 (1971).

[28] S. W. Hawking, Black holes in general relativity, Commun. Math. Phys. 25, 152 (1972).

[29] N. Gürlebeck, No-Hair Theorem for Black Holes in Astrophysical Environments, Phys. Rev. Lett. 114, 151102 (2015).

[30] L. Bildsten and C. Cutler, Tidal interactions of inspiraling compact binaries, Astrophys. J. 400, 175 (1992).

[31] A. Reisenegger and P. Goldreich, Excitation of neutron star normal modes during binary inspiral, Astrophys. J. 426, 688 (1994).

[32] D. Lai, F. A. Rasio, and S. L. Shapiro, Ellipsoidal figures of equilibrium - Compressible models, Astrophys. J. Suppl. 88, 205 (1993).

[33] D. Lai, Resonant oscillations and tidal heating in coalescing binary neutron stars, Mon. Not. R. Astron. Soc. 270, 611 (1994).

[34] J. P. Zahn, Reprint of 1977A\&A....57..383Z. Tidal friction in close binary stars, Astron. Astrophys. 500, 121 (1977).

[35] B. Willems, T. Van Hoolst, and P. Smeyers, Nonadiabatic resonant dynamic tides and orbital evolution in close binaries, Astron. Astrophys. 397, 973 (2003).

[36] J. P. Zahn, Forced oscillations in close binaries, the adiabatic approximation, Astron. Astrophys. 4, 452 (1970).

[37] Z. Kopal, Dynamics of Close Binary Systems (D. Reidel, Dordrecht, 1978).

[38] C. S. Kochanek, Coalescing binary neutron stars, Astrophys. J. 398, 234 (1992).

[39] D. Hansen, Dynamical evolution and leading order gravitational wave emission of Riemann-S binaries, Gen. Relativ. Gravit. 38, 1173 (2006). 
[40] T. Mora and C. M. Will, A Post-Newtonian diagnostic of quasiequilibrium binary configurations of compact objects, Phys. Rev. D 69, 104021 (2004); A Post-Newtonian diagnostic of quasiequilibrium binary configurations of compact objects, 71, 129901(E) (2005).

[41] K. D. Kokkotas and G. Schaefer, Tidal and tidal resonant effects in coalescing binaries, Mon. Not. R. Astron. Soc. 275, 301 (1995).

[42] E. E. Flanagan and T. Hinderer, Constraining neutron star tidal Love numbers with gravitational wave detectors, Phys. Rev. D 77, 021502 (2008).

[43] V. Ferrari, L. Gualtieri, and A. Maselli, Tidal interaction in compact binaries: A post-Newtonian affine framework, Phys. Rev. D 85, 044045 (2012).

[44] T. Damour, M. Soffel, and C.-m. Xu, General relativistic celestial mechanics. 2. Translational equations of motion, Phys. Rev. D 45, 1017 (1992).

[45] M. Shibata, Effects of tidal resonances in coalescing compact binary systems, Prog. Theor. Phys. 91, 871 (1994).

[46] Y. Rathore, A. E. Broderick, and R. Blandford, A variational formalism for tidal excitation: non-rotating, homentropic stars, Mon. Not. R. Astron. Soc. 339, 25 (2003).

[47] J. E. Vines and E. E. Flanagan, Post-1-Newtonian quadrupole tidal interactions in binary systems, Phys. Rev. D 88, 024046 (2013).

[48] J. Vines, E. E. Flanagan, and T. Hinderer, Post-1-Newtonian tidal effects in the gravitational waveform from binary inspirals, Phys. Rev. D 83, 084051 (2011).

[49] S. Chakrabarti, T. Delsate, and J. Steinhoff, Effective action and linear response of compact objects in Newtonian gravity, Phys. Rev. D 88, 084038 (2013).

[50] N. Andersson and W. C. G. Ho, Using gravitational-wave data to constrain dynamical tides in neutron star binaries, Phys. Rev. D 97, 023016 (2018).

[51] H. Yu and N. N. Weinberg, Resonant tidal excitation of superfluid neutron stars in coalescing binaries, Mon. Not. R. Astron. Soc. 464, 2622 (2017).

[52] D. Tsang, Shattering flares during close encounters of neutron stars, Astrophys. J. 777, 103 (2013).

[53] D. Tsang, J. S. Read, T. Hinderer, A. L. Piro, and R. Bondarescu, Resonant Shattering of Neutron Star Crusts, Phys. Rev. Lett. 108, 011102 (2012).

[54] P. Pnigouras, Gravitational-wave-driven tidal secular instability in neutron star binaries, Phys. Rev. D 100, 063016 (2019).

[55] G. Pratten, P. Schmidt, and T. Hinderer, Gravitational-wave asteroseismology with fundamental modes from compact binary inspirals, Nat. Commun. 11, 2553 (2020).

[56] P. Schmidt and T. Hinderer, Frequency domain model of $f$ mode dynamic tides in gravitational waveforms from compact binary inspirals, Phys. Rev. D 100, 021501 (2019).

[57] A. G. Suvorov and K. D. Kokkotas, Precursor flares of short gamma-ray bursts from crust yielding due to tidal resonances in coalescing binaries of rotating, magnetized neutron stars, Phys. Rev. D 101, 083002 (2020).

[58] N. Andersson and P. Pnigouras, Exploring the effective tidal deformability of neutron stars, Phys. Rev. D 101, 083001 (2020).

[59] Z. Pan, Z. Lyu, B. Bonga, N. Ortiz, and H. Yang, Probing Crust Meltdown in Inspiraling Binary Neutron Stars, Phys. Rev. Lett. 125, 201102 (2020).
[60] H. H.-Y. Ng, P. C.-K. Cheong, L.-M. Lin, and T. G. F. $\mathrm{Li}$, Gravitational-wave asteroseismology with f-modes from neutron star binaries at the merger phase, Astrophys. J. 915, 108 (2021).

[61] J. A. Pons, E. Berti, L. Gualtieri, G. Miniutti, and V. Ferrari, Gravitational signals emitted by a point mass orbiting a neutron star: Effects of stellar structure, Phys. Rev. D 65, 104021 (2002)..

[62] L. Gualtieri, E. Berti, J. A. Pons, G. Miniutti, and V. Ferrari, Gravitational signals emitted by a point mass orbiting a neutron star: A Perturbative approach, Phys. Rev. D 64, 104007 (2001).

[63] N. V. Krishnendu, K. G. Arun, and C. K. Mishra, Testing the Binary Black Hole Nature of A Compact Binary Coalescence, Phys. Rev. Lett. 119, 091101 (2017).

[64] A. Bohé, G. Faye, S. Marsat, and E. K. Porter, Quadraticin-spin effects in the orbital dynamics and gravitational-wave energy flux of compact binaries at the 3PN order, Classical Quantum Gravity 32, 195010 (2015).

[65] S. Marsat, Cubic order spin effects in the dynamics and gravitational wave energy flux of compact object binaries, Classical Quantum Gravity 32, 085008 (2015).

[66] M. Levi and J. Steinhoff, Spinning gravitating objects in the effective field theory in the post-Newtonian scheme, J. High Energy Phys. 09 (2015) 219.

[67] R. A. Porto, A. Ross, and I. Z. Rothstein, Spin induced multipole moments for the gravitational wave amplitude from binary inspirals to 2.5 Post-Newtonian order, J. Cosmol. Astropart. Phys. 09 (2012) 028.

[68] R. A. Porto, A. Ross, and I. Z. Rothstein, Spin induced multipole moments for the gravitational wave flux from binary inspirals to third Post-Newtonian order, J. Cosmol. Astropart. Phys. 03 (2011) 009.

[69] M. Levi and J. Steinhoff, Leading order finite size effects with spins for inspiralling compact binaries, J. High Energy Phys. 06 (2015) 059.

[70] A. Buonanno, G. Faye, and T. Hinderer, Spin effects on gravitational waves from inspiraling compact binaries at second post-Newtonian order, Phys. Rev. D 87, 044009 (2013).

[71] M. Levi and F. Teng, NLO gravitational quartic-in-spin interaction, J. High Energy Phys. 01 (2021) 066.

[72] M. Levi, A. J. Mcleod, and M. Von Hippel, NNNLO gravitational quadratic-in-spin interactions at the quartic order in $\mathrm{G}$, J. High Energy Phys. 07 (2021) 116.

[73] M. Levi, S. Mougiakakos, and M. Vieira, Gravitational cubicin-spin interaction at the next-to-leading post-Newtonian order, J. High Energy Phys. 01 (2021) 036.

[74] E. E. Flanagan and E. Racine, Gravitomagnetic resonant excitation of Rossby modes in coalescing neutron star binaries, Phys. Rev. D 75, 044001 (2007).

[75] M. Favata, Are neutron stars crushed? Gravitomagnetic tidal fields as a mechanism for binary-induced collapse, Phys. Rev. D 73, 104005 (2006).

[76] P. Landry and E. Poisson, Gravitomagnetic response of an irrotational body to an applied tidal field, Phys. Rev. D 91, 104026 (2015).

[77] P. Pani, L. Gualtieri, T. Abdelsalhin, and X. Jiménez-Forteza, Magnetic tidal Love numbers clarified, Phys. Rev. D 98, 124023 (2018). 
[78] P. Landry and E. Poisson, Dynamical response to a stationary tidal field, Phys. Rev. D 92, 124041 (2015).

[79] B. Banihashemi and J. Vines, Gravitomagnetic tidal effects in gravitational waves from neutron star binaries, Phys. Rev. D 101, 064003 (2020).

[80] E. Poisson, Gravitomagnetic tidal resonance in neutron-star binary inspirals, Phys. Rev. D 101, 104028 (2020).

[81] E. Poisson, Gravitomagnetic Love tensor of a slowly rotating body: Post-Newtonian theory, Phys. Rev. D 102, 064059 (2020).

[82] E. Poisson and C. Buisson, Tidal driving of inertial modes of Maclaurin spheroids, Phys. Rev. D 102, 104005 (2020).

[83] P. K. Gupta, J. Steinhoff, and T. Hinderer, Relativistic effective action of dynamical gravitomagnetic tides for slowly rotating neutron stars, Phys. Rev. Research 3, 013147 (2021).

[84] R. Gold, S. Bernuzzi, M. Thierfelder, B. Brugmann, and F. Pretorius, Eccentric binary neutron star mergers, Phys. Rev. D 86, 121501 (2012).

[85] C. Chirenti, R. Gold, and M. C. Miller, Gravitational waves from f-modes excited by the inspiral of highly eccentric neutron star binaries, Astrophys. J. 837, 67 (2017).

[86] H. Yang, Inspiralling eccentric binary neutron stars: Orbital motion and tidal resonance, Phys. Rev. D 100, 064023 (2019).

[87] M. Vick and D. Lai, Tidal effects in eccentric coalescing neutron star binaries, Phys. Rev. D 100, 063001 (2019).

[88] W. Xu and D. Lai, Resonant tidal excitation of oscillation modes in merging binary neutron stars: Inertial-gravity modes, Phys. Rev. D 96, 083005 (2017).

[89] R. Essick, S. Vitale, and N. N. Weinberg, Impact of the tidal $\mathrm{p}$-g instability on the gravitational wave signal from coalescing binary neutron stars, Phys. Rev. D 94, 103012 (2016).

[90] X. Jiménez Forteza, T. Abdelsalhin, P. Pani, and L. Gualtieri, Impact of high-order tidal terms on binary neutron-star waveforms, Phys. Rev. D 98, 124014 (2018).

[91] T. Abdelsalhin, L. Gualtieri, and P. Pani, Post-Newtonian spintidal couplings for compact binaries, Phys. Rev. D 98, 104046 (2018).

[92] P. Pani, L. Gualtieri, and V. Ferrari, Tidal Love numbers of a slowly spinning neutron star, Phys. Rev. D 92, 124003 (2015).

[93] P. Pani, L. Gualtieri, A. Maselli, and V. Ferrari, Tidal deformations of a spinning compact object, Phys. Rev. D 92, 024010 (2015).

[94] S. Endlich and R. Penco, Effective field theory approach to tidal dynamics of spinning astrophysical systems, Phys. Rev. D 93, 064021 (2016).

[95] J. Gagnon-Bischoff, S. R. Green, P. Landry, and N. Ortiz, Extended I-Love relations for slowly rotating neutron stars, Phys. Rev. D 97, 064042 (2018).

[96] P. Landry, Tidal deformation of a slowly rotating material body: Interior metric and Love numbers, Phys. Rev. D 95, 124058 (2017).

[97] P. Landry and E. Poisson, Tidal deformation of a slowly rotating material body. External metric, Phys. Rev. D 91, 104018 (2015).

[98] T. Dietrich, A. Samajdar, S. Khan, N. K. Johnson-McDaniel, R. Dudi, and W. Tichy, Improving the NRTidal model for binary neutron star systems, Phys. Rev. D 100, 044003 (2019).

[99] A. Le Tiec and M. Casals, Spinning Black Holes Fall in Love, Phys. Rev. Lett. 126, 131102 (2021).
[100] A. Le Tiec, M. Casals, and E. Franzin, Tidal love numbers of kerr black holes, Phys. Rev. D 103, 084021 (2021).

[101] H. S. Chia, Tidal deformation and dissipation of rotating black holes, Phys. Rev. D 104, 024013 (2021).

[102] W. D. Goldberger, J. Li, and I. Z. Rothstein, Non-conservative effects on Spinning Black Holes from World-Line Effective Field Theory, J. High Energy Phys. 06 (2021) 053.

[103] W. C. G. Ho and D. Lai, Resonant tidal excitations of rotating neutron stars in coalescing binaries, Mon. Not. R. Astron. Soc. 308, 153 (1999).

[104] S. Ma, H. Yu, and Y. Chen, Excitation of f-modes during mergers of spinning binary neutron star, Phys. Rev. D 101, 123020 (2020).

[105] D. Lai, Secular instability of g modes in rotating neutron stars, Mon. Not. R. Astron. Soc. 307, 1001 (1999).

[106] D. Lai, Dynamical tides in rotating binary stars, Astrophys. J. 490, 847 (1997).

[107] P. B. Ivanov, J. C. B. Papaloizou, and S. V. Chernov, A new general normal mode approach to dynamic tides in rotating stars with realistic structure and its applications, Astron. Astrophys. Trans. 28, 355 (2014).

[108] D. Lai and Y. Wu, Resonant tidal excitations of inertial modes in coalescing neutron star binaries, Phys. Rev. D 74, 024007 (2006).

[109] S. Ma, H. Yu, and Y. Chen, Revisiting the tidal excitation of Rossby modes in coalescing binary systems, Phys. Rev. D 103, 063020 (2021).

[110] G. Kälin and R. A. Porto, Post-Minkowskian Effective Field Theory for Conservative Binary Dynamics, J. High Energy Phys. 11 (2020) 106.

[111] G. Kälin, Z. Liu, and R. A. Porto, Conservative tidal effects in compact binary systems to next-to-leading post-minkowskian order, Phys. Rev. D 102, 124025 (2020).

[112] D. Bini, T. Damour, and A. Geralico, Scattering of tidally interacting bodies in post-Minkowskian gravity, Phys. Rev. D 101, 044039 (2020).

[113] C. Cheung and M. P. Solon, Tidal Effects in the PostMinkowskian Expansion, Phys. Rev. Lett. 125, 191601 (2020).

[114] K. Haddad and A. Helset, Tidal effects in quantum field theory, J. High Energy Phys. 12 (2020) 024.

[115] C. Cheung, N. Shah, and M. P. Solon, Mining the geodesic equation for scattering data, Phys. Rev. D 103, 024030 (2021).

[116] Z. Bern, J. Parra-Martinez, R. Roiban, E. Sawyer, and C.-H. Shen, Leading nonlinear tidal effects and scattering amplitudes, J. High Energy Phys. 05 (2021) 188.

[117] P. Ajith et al., Phenomenological template family for blackhole coalescence waveforms, Classical Quantum Gravity 24, S689 (2007).

[118] P. Ajith et al., A Template bank for gravitational waveforms from coalescing binary black holes. I. Non-spinning binaries, Phys. Rev. D 77, 104017 (2008); A Template bank for gravitational waveforms from coalescing binary black holes. I. Non-spinning binaries, 79, 129901(E) (2009)].

[119] S. Khan, S. Husa, M. Hannam, F. Ohme, M. Pürrer, X. Jiménez Forteza, and A. Bohé, Frequency-domain gravitational waves from nonprecessing black-hole binaries. II. A phenomenological model for the advanced detector era, Phys. Rev. D 93, 044007 (2016). 
[120] S. Husa, S. Khan, M. Hannam, M. Pürrer, F. Ohme, X. Jiménez Forteza, and A. Bohé, Frequency-domain gravitational waves from nonprecessing black-hole binaries. I. New numerical waveforms and anatomy of the signal, Phys. Rev. D 93, 044006 (2016).

[121] M. Hannam, P. Schmidt, A. Bohé, L. Haegel, S. Husa, F. Ohme, G. Pratten, and M. Pürrer, Simple Model of Complete Precessing Black-Hole-Binary Gravitational Waveforms, Phys. Rev. Lett. 113, 151101 (2014).

[122] P. Schmidt, M. Hannam, and S. Husa, Towards models of gravitational waveforms from generic binaries: A simple approximate mapping between precessing and nonprecessing inspiral signals, Phys. Rev. D 86, 104063 (2012).

[123] P. Schmidt, F. Ohme, and M. Hannam, Towards models of gravitational waveforms from generic binaries II: Modelling precession effects with a single effective precession parameter, Phys. Rev. D 91, 024043 (2015).

[124] P. Ajith et al., Inspiral-Merger-Ringdown Waveforms for Black-Hole Binaries with Non-Precessing Spins, Phys. Rev. Lett. 106, 241101 (2011).

[125] L. Santamaria et al., Matching post-Newtonian and numerical relativity waveforms: Systematic errors and a new phenomenological model for non-precessing black hole binaries, Phys. Rev. D 82, 064016 (2010).

[126] S. Khan, F. Ohme, K. Chatziioannou, and M. Hannam, Including higher order multipoles in gravitational-wave models for precessing binary black holes, Phys. Rev. D 101, 024056 (2020).

[127] C. García-Quirós, M. Colleoni, S. Husa, H. Estellés, G. Pratten, A. Ramos-Buades, M. Mateu-Lucena, and R. Jaume, Multimode frequency-domain model for the gravitational wave signal from nonprecessing black-hole binaries, Phys. Rev. D 102, 064002 (2020).

[128] T. Dietrich, S. Bernuzzi, and W. Tichy, Closed-form tidal approximants for binary neutron star gravitational waveforms constructed from high-resolution numerical relativity simulations, Phys. Rev. D 96, 121501 (2017).

[129] T. Dietrich et al., Matter imprints in waveform models for neutron star binaries: Tidal and self-spin effects, Phys. Rev. D 99, 024029 (2019).

[130] G. Pratten, S. Husa, C. Garcia-Quiros, M. Colleoni, A. RamosBuades, H. Estelles, and R. Jaume, Setting the cornerstone for a family of models for gravitational waves from compact binaries: The dominant harmonic for nonprecessing quasicircular black holes, Phys. Rev. D 102, 064001 (2020).

[131] G. Pratten et al., Let's twist again: Computationally efficient models for the dominant and sub-dominant harmonic modes of precessing binary black holes, Phys. Rev. D 103, 104056 (2021).

[132] A. Buonanno and T. Damour, Effective one-body approach to general relativistic two-body dynamics, Phys. Rev. D 59, 084006 (1999).

[133] A. Buonanno and T. Damour, Transition from inspiral to plunge in binary black hole coalescences, Phys. Rev. D 62, 064015 (2000).

[134] A. Bohé et al., Improved effective-one-body model of spinning, nonprecessing binary black holes for the era of gravitational-wave astrophysics with advanced detectors, Phys. Rev. D 95, 044028 (2017).
[135] S. Babak, A. Taracchini, and A. Buonanno, Validating the effective-one-body model of spinning, precessing binary black holes against numerical relativity, Phys. Rev. D 95, 024010 (2017).

[136] A. Taracchini, Y. Pan, A. Buonanno, E. Barausse, M. Boyle, T. Chu, G. Lovelace, H. P. Pfeiffer, and M. A. Scheel, Prototype effective-one-body model for nonprecessing spinning inspiralmerger-ringdown waveforms, Phys. Rev. D 86, 024011 (2012).

[137] A. Taracchini et al., Effective-one-body model for black-hole binaries with generic mass ratios and spins, Phys. Rev. D 89, 061502 (2014).

[138] Y. Pan, A. Buonanno, A. Taracchini, L. E. Kidder, A. H. Mroué, H. P. Pfeiffer, M. A. Scheel, and B. Szilágyi, Inspiral-merger-ringdown waveforms of spinning, precessing black-hole binaries in the effective-one-body formalism, Phys. Rev. D 89, 084006 (2014).

[139] Y. Pan, A. Buonanno, A. Taracchini, M. Boyle, L. E. Kidder, A. H. Mroué, H. P. Pfeiffer, M. A. Scheel, B. Szilágyi, and A. Zenginoglu, Stability of nonspinning effective-one-body model in approximating two-body dynamics and gravitationalwave emission, Phys. Rev. D 89, 061501 (2014).

[140] E. Barausse and A. Buonanno, An Improved effective-onebody Hamiltonian for spinning black-hole binaries, Phys. Rev. D 81, 084024 (2010).

[141] E. Barausse, E. Racine, and A. Buonanno, Hamiltonian of a spinning test-particle in curved spacetime, Phys. Rev. D 80, 104025 (2009); Hamiltonian of a spinning test-particle in curved spacetime, 85, 069904(E) (2012).

[142] E. Barausse and A. Buonanno, Extending the effective-onebody Hamiltonian of black-hole binaries to include next-tonext-to-leading spin-orbit couplings, Phys. Rev. D 84, 104027 (2011).

[143] Y. Pan, A. Buonanno, M. Boyle, L. T. Buchman, L. E. Kidder, H. P. Pfeiffer, and M. A. Scheel, Inspiral-mergerringdown multipolar waveforms of nonspinning black-hole binaries using the effective-one-body formalism, Phys. Rev. D 84, 124052 (2011).

[144] R. Cotesta, A. Buonanno, A. Bohé, A. Taracchini, I. Hinder, and S. Ossokine, Enriching the symphony of gravitational waves from binary black holes by tuning higher harmonics, Phys. Rev. D 98, 084028 (2018).

[145] S. Ossokine et al., Multipolar effective-one-body waveforms for precessing binary black holes: Construction and validation, Phys. Rev. D 102, 044055 (2020).

[146] A. Nagar, F. Messina, P. Rettegno, D. Bini, T. Damour, A. Geralico, S. Akcay, and S. Bernuzzi, Nonlinear-in-spin effects in effective-one-body waveform models of spin-aligned, inspiralling, neutron star binaries, Phys. Rev. D 99, 044007 (2019).

[147] A. Nagar et al., Time-domain effective-one-body gravitational waveforms for coalescing compact binaries with nonprecessing spins, tides and self-spin effects, Phys. Rev. D 98, 104052 (2018).

[148] T. Damour and A. Nagar, New effective-one-body description of coalescing nonprecessing spinning black-hole binaries, Phys. Rev. D 90, 044018 (2014).

[149] T. Damour and A. Nagar, A new analytic representation of the ringdown waveform of coalescing spinning black hole binaries, Phys. Rev. D 90, 024054 (2014). 
[150] T. Damour, A. Nagar, and S. Bernuzzi, Improved effectiveone-body description of coalescing nonspinning black-hole binaries and its numerical-relativity completion, Phys. Rev. D 87, 084035 (2013).

[151] T. Damour and A. Nagar, An Improved analytical description of inspiralling and coalescing black-hole binaries, Phys. Rev. D 79, 081503 (2009).

[152] T. Damour, A. Nagar, M. Hannam, S. Husa, and B. Bruegmann, Accurate Effective-One-Body waveforms of inspiralling and coalescing black-hole binaries, Phys. Rev. D 78, 044039 (2008)

[153] T. Damour, B. R. Iyer, and A. Nagar, Improved resummation of post-Newtonian multipolar waveforms from circularized compact binaries, Phys. Rev. D 79, 064004 (2009).

[154] T. Damour, A. Nagar, E. N. Dorband, D. Pollney, and L. Rezzolla, Faithful effective-one-body waveforms of equalmass coalescing black-hole binaries, Phys. Rev. D 77, 084017 (2008).

[155] F. Messina, A. Maldarella, and A. Nagar, Factorization and resummation: A new paradigm to improve gravitational wave amplitudes. II: The higher multipolar modes, Phys. Rev. D 97, 084016 (2018).

[156] A. Nagar, G. Riemenschneider, and G. Pratten, Impact of numerical relativity information on effective-one-body waveform models, Phys. Rev. D 96, 084045 (2017).

[157] A. Nagar and A. Shah, Factorization and resummation: A new paradigm to improve gravitational wave amplitudes, Phys. Rev. D 94, 104017 (2016).

[158] T. Damour, Coalescence of two spinning black holes: an effective one-body approach, Phys. Rev. D 64, 124013 (2001).

[159] T. Damour, P. Jaranowski, and G. Schaefer, Effective one body approach to the dynamics of two spinning black holes with next-to-leading order spin-orbit coupling, Phys. Rev. D 78, 024009 (2008).

[160] A. Nagar, Effective one body Hamiltonian of two spinning black-holes with next-to-next-to-leading order spin-orbit coupling, Phys. Rev. D 84, 084028 (2011); Effective one body Hamiltonian of two spinning black-holes with next-to-next-toleading order spin-orbit coupling, 88, 089901(E) (2013).

[161] S. Balmelli and P. Jetzer, Effective-one-body Hamiltonian with next-to-leading order spin-spin coupling for two nonprecessing black holes with aligned spins, Phys. Rev. D 87, 124036 (2013); Effective-one-body Hamiltonian with next-toleading order spin-spin coupling for two nonprecessing black holes with aligned spins, 90, 089905(E) (2014).

[162] A. Nagar, G. Pratten, G. Riemenschneider, and R. Gamba, Multipolar effective one body model for nonspinning black hole binaries, Phys. Rev. D 101, 024041 (2020).

[163] S. Bernuzzi, A. Nagar, and A. Zenginoglu, Horizon-absorption effects in coalescing black-hole binaries: An effective-onebody study of the non-spinning case, Phys. Rev. D 86, 104038 (2012).

[164] A. Nagar and S. Akcay, Horizon-absorbed energy flux in circularized, nonspinning black-hole binaries and its effectiveone-body representation, Phys. Rev. D 85, 044025 (2012).

[165] T. Dietrich, T. Hinderer, and A. Samajdar, Interpreting binary neutron star mergers: Describing the binary neutron star dynamics, modelling gravitational waveforms, and analyzing detections, Gen. Relativ. Gravit. 53, 27 (2021).
[166] T. Damour and A. Nagar, The effective-one-body approach to the general relativistic two body problem, Lect. Notes Phys. 905, 273 (2016).

[167] T. Damour and A. Nagar, The Effective One body description of the two-body problem, mass and motion in general relativity, Proceedings, School on Mass, Orleans, France, June 23-25, 2008, Fundam. Theor. Phys. 162, 211 (2011), arXiv:0906.1769 [gr-qc].

[168] A. Buonanno and B. S. Sathyaprakash, Sources of Gravitational Waves: Theory and Observations (Cambridge University Press, Cambridge, 2014), pp. 287-346.

[169] T. Damour, The general relativistic two body problem and the effective one body formalism, Fundam. Theor. Phys. 177, 111 (2014).

[170] M. Hannam, Modelling gravitational waves from precessing black-hole binaries: Progress, challenges and prospects, Gen. Relativ. Gravit. 46, 1767 (2014).

[171] T. Damour, Introductory lectures on the effective one body formalism, Int. J. Mod. Phys. A23, 1130 (2008)

[172] T. Hinderer et al., Effects of Neutron-Star Dynamic Tides on Gravitational Waveforms within the Effective-One-Body Approach, Phys. Rev. Lett. 116, 181101 (2016).

[173] J. Steinhoff, T. Hinderer, A. Buonanno, and A. Taracchini, Dynamical tides in general relativity: Effective action and effective-one-body hamiltonian, Phys. Rev. D 94, 104028 (2016).

[174] F. Foucart et al., Gravitational waveforms from spectral Einstein code simulations: Neutron star-neutron star and lowmass black hole-neutron star binaries, Phys. Rev. D 99, 044008 (2019).

[175] J. Aasi et al. (LIGO Scientific), Advanced LIGO, Classical Quantum Gravity 32, 074001 (2015).

[176] F. Acernese et al. (VIRGO), Advanced virgo: A second-generation interferometric gravitational wave detector, Classical Quantum Gravity 32, 024001 (2015).

[177] T. Akutsu et al. (KAGRA), Overview of KAGRA: Detector design and construction history, arXiv:2005.05574.

[178] S. Chandrasekhar, A general variational principle governing the radial and the non-radial oscillations of gaseous masses. Astrophys. J. 139, 664 (1964).

[179] A. K. Schenk, P. Arras, E. E. Flanagan, S. A. Teukolsky, and I. Wasserman, Nonlinear mode coupling in rotating stars and the r mode instability in neutron stars, Phys. Rev. D 65, 024001 (2002).

[180] D. Lynden-Bell and J. P. Ostriker, On the stability of differentially rotating bodies, Mon. Not. R. Astron. Soc. 136, 293 (1967).

[181] R. A. Porto, Post-Newtonian corrections to the motion of spinning bodies in NRGR, Phys. Rev. D 73, 104031 (2006).

[182] W. D. Goldberger and A. Ross, Gravitational radiative corrections from effective field theory, Phys. Rev. D 81, 124015 (2010).

[183] M. Levi, Next to leading order gravitational spin-orbit coupling in an effective field theory approach, Phys. Rev. D 82, 104004 (2010).

[184] W. D. Goldberger and I. Z. Rothstein, An Effective field theory of gravity for extended objects, Phys. Rev. D 73, 104029 (2006).

[185] W. D. Goldberger, Les Houches lectures on effective field theories and gravitational radiation, in Les Houches Summer 
School, Session 86: Particle Physics and Cosmology: The Fabric of Spacetime, Les Houches, France, July 31-August 25, 2006 (Elsevier, Amsterdam, 2007).

[186] S. Foffa and R. Sturani, Effective field theory methods to model compact binaries, Classical Quantum Gravity 31, 043001 (2014).

[187] I. Z. Rothstein, Progress in effective field theory approach to the binary inspiral problem, Gen. Relativ. Gravit. 46, 1726 (2014).

[188] R. A. Porto, The effective field theorist's approach to gravitational dynamics, Phys. Rep. 633, 1 (2016).

[189] M. Levi, Effective field theories of post-newtonian gravity: A comprehensive review, Rep. Prog. Phys. 83, 075901 (2020).

[190] S. Chakrabarti, T. Delsate, and J. Steinhoff, New perspectives on neutron star and black hole spectroscopy and dynamic tides, arXiv:1304.2228.

[191] D. Bini, T. Damour, and G. Faye, Effective action approach to higher-order relativistic tidal interactions in binary systems and their effective one body description, Phys. Rev. D 85 , 124034 (2012).

[192] Q. Henry, G. Faye, and L. Blanchet, Tidal effects in the equations of motion of compact binary systems to nextto-next-to-leading post-Newtonian order, Phys. Rev. D 101, 064047 (2020).

[193] Q. Henry, G. Faye, and L. Blanchet, Tidal effects in the gravitational-wave phase evolution of compact binary systems to next-to-next-to-leading post-Newtonian order, Phys. Rev. D 102, 044033 (2020).

[194] Q. Henry, G. Faye, and L. Blanchet, Hamiltonian for tidal interactions in compact binary systems to next-to-next-toleading post-Newtonian order, Phys. Rev. D 102, 124074 (2020).

[195] J. B. Hartle, Slowly rotating relativistic stars. I. Equations of structure, Astrophys. J. 150, 1005 (1967).

[196] E. Poisson, Gravitational waves from inspiraling compact binaries: The Quadrupole moment term, Phys. Rev. D 57, 5287 (1998).

[197] W. G. Laarakkers and E. Poisson, Quadrupole moments of rotating neutron stars, Astrophys. J. 512, 282 (1999).

[198] M. Khalil, J. Steinhoff, J. Vines, and A. Buonanno, Fourth post-Newtonian effective-one-body Hamiltonians with generic spins, Phys. Rev. D 101, 104034 (2020).

[199] M. Levi and J. Steinhoff, Complete conservative dynamics for inspiralling compact binaries with spins at fourth postNewtonian order, arXiv:1607.04252.

[200] G. Schaefer, Gravitomagnetic effects, HYPER Symposium Paris, France, November 4-6, 2002, Gen. Relativ. Gravit. 36, 2223 (2004).

[201] D. D. Doneva, E. Gaertig, K. D. Kokkotas, and C. Krüger, Gravitational wave asteroseismology of fast rotating neutron stars with realistic equations of state, Phys. Rev. D 88, 044052 (2013).

[202] E. Gaertig and K. D. Kokkotas, Oscillations of rapidly rotating relativistic stars, Phys. Rev. D 78, 064063 (2008).

[203] E. Gaertig and K. D. Kokkotas, Gravitational wave asteroseismology with fast rotating neutron stars, Phys. Rev. D 83, 064031 (2011).

[204] C. J. Krüger and K. D. Kokkotas, Fast Rotating Relativistic Stars: Spectra and Stability without Approximation, Phys. Rev. Lett. 125, 111106 (2020).
[205] N. Stergioulas and S. Morsink, RNS code, http://www.gravity. phys.uwm.edu/rns/.

[206] N. Stergioulas and J. Friedman, Comparing models of rapidly rotating relativistic stars constructed by two numerical methods, Astrophys. J. 444, 306 (1995).

[207] H. Yu, N. N. Weinberg, and P. Arras, Tides in the higheccentricity migration of hot Jupiters: Triggering diffusive growth by nonlinear mode interactions, arXiv:2104.04929.

[208] U. Lee and T. E. Strohmayer, Nonradial oscillations of rotating neutron stars: The effects of the Coriolis force, Astron. Astrophys. 311, 155 (1996).

[209] K. Yagi and N. Yunes, Approximate universal relations for neutron stars and quark stars, Phys. Rep. 681, 1 (2017).

[210] S. V. Chaurasia, T. Dietrich, N. K. Johnson-McDaniel, M. Ujevic, W. Tichy, and B. Brügmann, Gravitational waves and mass ejecta from binary neutron star mergers: Effect of large eccentricities, Phys. Rev. D 98, 104005 (2018).

[211] L. D. Landau and E. M. Lifshitz, Mechanics, Third Edition: Volume 1 (Course of Theoretical Physics) (ButterworthHeinemann, Oxford, 1976).

[212] "Spectral Einstein Code," https://www.black-holes.org/code/ SpEC.html.

[213] M. D. Duez, F. Foucart, L. E. Kidder, H. P. Pfeiffer, M. A. Scheel, and S. A. Teukolsky, Evolving black hole-neutron star binaries in general relativity using pseudospectral and finite difference methods, Phys. Rev. D 78, 104015 (2008).

[214] F. Foucart, M. B. Deaton, M. D. Duez, L. E. Kidder, I. MacDonald, C. D. Ott, H. P. Pfeiffer, M. A. Scheel, B. Szilagyi, and S. A. Teukolsky, Black hole-neutron star mergers at realistic mass ratios: Equation of state and spin orientation effects, Phys. Rev. D 87, 084006 (2013).

[215] B. Bruegmann, J. A. Gonzalez, M. Hannam, S. Husa, U. Sperhake, and W. Tichy, Calibration of moving puncture simulations, Phys. Rev. D 77, 024027 (2008).

[216] M. Thierfelder, S. Bernuzzi, and B. Bruegmann, Numerical relativity simulations of binary neutron stars, Phys. Rev. D 84, 044012 (2011).

[217] T. Dietrich, S. Bernuzzi, M. Ujevic, and B. Brügmann, Numerical relativity simulations of neutron star merger remnants using conservative mesh refinement, Phys. Rev. D 91, 124041 (2015).

[218] M. Boyle et al., The SXS Collaboration catalog of binary black hole simulations, Classical Quantum Gravity 36, 195006 (2019)

[219] F. Foucart, L. E. Kidder, H. P. Pfeiffer, and S. A. Teukolsky, Initial data for black hole-neutron star binaries: A Flexible, high-accuracy spectral method, Phys. Rev. D 77, 124051 (2008).

[220] H. P. Pfeiffer, D. A. Brown, L. E. Kidder, L. Lindblom, G. Lovelace, and M. A. Scheel, Reducing orbital eccentricity in binary black hole simulations, Classical Quantum Gravity 24, S59 (2007).

[221] A. Matas et al., Aligned-spin neutron-star-black-hole waveform model based on the effective-one-body approach and numerical-relativity simulations, Phys. Rev. D 102, 043023 (2020).

[222] J. S. Read, B. D. Lackey, B. J. Owen, and J. L. Friedman, Constraints on a phenomenologically parameterized neutron-star equation of state, Phys. Rev. D 79, 124032 (2009). 
[223] T. Dietrich, S. Bernuzzi, B. Bruegmann, and W. Tichy, Highresolution numerical relativity simulations of spinning binary neutron star mergers, in Proceedings of the 26th Euromicro International Conference on Parallel, Distributed and Network-based Processing (PDP 2018): Cambridge, UK, March 21-23, 2018 (IEEE Computer Society, Washington, DC, 2018), pp. 682-689.

[224] T. Dietrich, D. Radice, S. Bernuzzi, F. Zappa, A. Perego, B. Brügmann, S. V. Chaurasia, R. Dudi, W. Tichy, and M.
Ujevic, CoRe database of binary neutron star merger waveforms, Classical Quantum Gravity 35, 24LT01 (2018).

[225] S. Bernuzzi and T. Dietrich, Gravitational waveforms from binary neutron star mergers with high-order weightedessentially-nonoscillatory schemes in numerical relativity, Phys. Rev. D 94, 064062 (2016).

[226] R. Prix and M. L. E. Rieutord, Adiabatic oscillations of nonrotating superfluid neutron stars, Astron. Astrophys. 393, 949 (2002). 